\title{
Approximation Algorithms for Stochastic Submodular Set Cover with Applications to Boolean Function Evaluation and Min-Knapsack
}

\author{
AMOL DESHPANDE, University of Maryland \\ LISA HELLERSTEIN, NYU School of Engineering \\ DEVORAH KLETENIK, Brooklyn College, City University of New York
}

\begin{abstract}
We present a new approximation algorithm for the stochastic submodular set cover (SSSC) problem called adaptive dual greedy. We use this algorithm to obtain a 3-approximation algorithm solving the stochastic Boolean function evaluation (SBFE) problem for linear threshold formulas (LTFs). We also obtain a 3approximation algorithm for the closely related stochastic min-knapsack problem and a 2 -approximation for a variant of that problem.

We prove a new approximation bound for a previous algorithm for the SSSC problem, the adaptive greedy algorithm of Golovin and Krause.

We also consider an approach to approximating SBFE problems using the adaptive greedy algorithm, which we call the $Q$-value approach. This approach easily yields a new result for evaluation of CDNF (conjunctive / disjunctive normal form) formulas, and we apply variants of it to simultaneous evaluation problems and a ranking problem. However, we show that the $Q$-value approach provably cannot be used to obtain a sublinear approximation factor for the SBFE problem for LTFs or read-once disjunctive normal form formulas.
\end{abstract}

CCS Concepts: - Theory of computation $\rightarrow$ Approximation algorithms analysis; Packing and covering problems; Stochastic approximation; Database query processing and optimization (theory); - Computing methodologies $\rightarrow$ Representation of Boolean functions; Cost-sensitive learning

Additional Key Words and Phrases: Sequential testing, Boolean function evaluation

ACM Reference Format:

Amol Deshpande, Lisa Hellerstein, and Devorah Kletenik. 2016. Approximation algorithms for stochastic submodular set cover with applications to Boolean function evaluation and min-knapsack. ACM Trans. Algorithms 12, 3, Article 42 (April 2016), 28 pages.

DOI: http://dx.doi.org/10.1145/2876506

\section{INTRODUCTION}

We present new results on the stochastic submodular set cover (SSSC) problem, introduced by Golovin and Krause [2011]. This problem generalizes the classical NPcomplete set cover problem. We also present related results on the stochastic Boolean function evaluation (SBFE) problem, as well as stochastic min-knapsack.

L. Hellerstein was partially supported by NSF grants 1217968 and 0917153 . D. Kletenik was partially supported by NSF grant 0917153 and by U.S. Department of Education GAANN grant P200A090157. A. Deshpande was partially supported by NSF grants 0916736 and 1218367.

Authors' addresses: A. Deshpande, 3221 A. V. Williams Bldg., Department of Computer Science, University of Maryland, College Park, MD 20742; email: amol@cs.umd.edu; L. Hellerstein, Department of Computer Science and Engineering, NYU School of Engineering, 2 Metrotech Center, Brooklyn, NY 11201; email: lisa.hellerstein@nyu.edu; D. Kletenik, Department of Computer and Information Science, Brooklyn College, City University of New York, 2900 Bedford Avenue, Brooklyn, NY 11210; email: kletenik@sci.brooklyn.cuny.edu.

Permission to make digital or hard copies of part or all of this work for personal or classroom use is granted without fee provided that copies are not made or distributed for profit or commercial advantage and that copies show this notice on the first page or initial screen of a display along with the full citation. Copyrights for components of this work owned by others than ACM must be honored. Abstracting with credit is permitted. To copy otherwise, to republish, to post on servers, to redistribute to lists, or to use any component of this work in other works requires prior specific permission and/or a fee. Permissions may be requested from Publications Dept., ACM, Inc., 2 Penn Plaza, Suite 701, New York, NY 10121-0701 USA, fax +1 (212) 869-0481, or permissions@acm.org.

(c) 2016 ACM 1549-6325/2016/04-ART42 $\$ 15.00$

DOI: http://dx.doi.org/10.1145/2876506 
The classical set cover problem takes as input a finite ground set $X$; a set $F=$ $\left\{S_{1}, \ldots, S_{m}\right\}$, where each $S_{i} \subseteq X$; and a cost vector $c=\left(c_{1}, \ldots, c_{n}\right)$, where each $c_{i} \geq 0$. A subset $F^{\prime} \subseteq F$, where $\bigcup_{S_{i} \in F^{\prime}} S_{j}=X$ such that the cost $\sum_{S_{i} \in F^{\prime}} c_{j}$ of $F^{\prime}$ is minimized.

As a concrete example of the classical set cover problem, consider a scenario where we have a set $X$ of locations that we need to monitor and have sensors, numbered 1 through $n$, near those locations. Sensor $i$ can monitor only a subset $S_{i}$ of the locations in $X$, and taking a reading from sensor $i$ has a cost of $c_{i}$. We want to take readings from a subset $F^{\prime}$ of the sensors that together monitor all of the locations in $X$ at minimum cost.

Now suppose that each sensor could be either working or faulty, and that the locations it can monitor will be different according to the state of that sensor. For example, if sensor $i$ is working, it covers one set of locations $S_{i}$, but if it is faulty, it covers another set $S_{i}^{\prime}$. In addition, suppose that we will not discover the state of sensor $i$ (and the locations it monitors) until we take a reading from it. We may then want to choose which sensors to read sequentially, as the knowledge that one sensor is faulty can be useful in deciding which other sensor we should choose next. This type of scenario gives rise to a stochastic version of the classical set cover problem studied by Goemans and Vondrák [2006]. In this version, instead of subsets $S_{i}$, we have "items" $i$, and each item $i$ has a random state, which is drawn from a known distribution that is given as part of the input. The states of different items are independent. The subset of ground set $X$ covered by item $i$ depends on the state of item $i$, and items are chosen sequentially and adaptively until all elements of $X$ are covered. The problem is to choose the items so as to minimize their expected cost.

A different generalization of the classical set cover problem addresses a more abstract task. In the submodular set cover (SSC) problem, studied by Wolsey [1982], the input consists of a set $N=\{1, \ldots, n\}$ of items; a monotone, submodular utility function $g$ assigning a nonnegative value (utility) to each subset of $N$; and a cost vector $c=$ $\left(c_{1}, \ldots, c_{n}\right)$, where each $c_{i} \geq 0$. The problem is to find a subset $N^{\prime}$ of $N$ such that $g\left(N^{\prime}\right)=g(N)$ and $\sum_{i \in N^{\prime}} c_{i}$ is minimized. W call $N^{\prime}$ a cover. The connection to classical set cover is seen if we make the items correspond to the subsets $S_{i}$ and define the value of utility function $g$ on a subset of the items $i$ to be the number of elements of the ground set that are covered by the corresponding $S_{i}$.

The SSSC problem addressed in this article is a combination of the preceding two generalizations. ${ }^{1}$ There is a set $N=\{1, \ldots, n\}$ of items. Each item has an initially unknown state that is randomly chosen from a finite state set according to a known distribution. The states of different items are independent. There is a monotone, submodular utility function $g$ assigning a value to each subset of the items. In general, this value can depend both on which items are in the subset and on the states of those items. The exception is that the value of $g$ on the set of all items in $N$ must be equal to some fixed value $Q \geq 0$, independent of the item states. We call $Q$ the "goal" utility. The problem is to choose the items sequentially and adaptively until their utility is at least $Q$ so that the expected cost of the chosen items is minimized.

Golovin and Krause presented and analyzed an algorithm for the SSSC problem called adaptive greedy.

Overview of main results. We present a new algorithm for the SSSC problem that we call adaptive dual greedy (henceforth, ADG). Using this algorithm, we obtain a 3-approximation algorithm solving the SBFE problem for linear threshold formulas (LTFs). We also obtain a 3-approximation for the stochastic min-knapsack problem and a 2 -approximation for a variant of that problem.

\footnotetext{
${ }^{1}$ Golovin and Krause called the problem stochastic submodular coverage, not stochastic submdodular set cover, because the cover is not formed using sets. Our choice of name is for consistency with terminology of Fujito [2000].
} 
We prove new bounds on the adaptive greedy algorithm.

We also consider an approach to approximating SBFE problems, using the adaptive greedy algorithm, that we call the $Q$-value approach. The approach easily yields a new result for evaluation of CDNF (conjunctive / disjunctive normal form) formulas, and we apply variants of it to simultaneous evaluation problems and a ranking problem. However, we show that the $Q$-value approach provably cannot be used to obtain a sublinear approximation factor for the SBFE problem for LTFs or read-once disjunctive normal form (DNF) formulas.

We note that our work on SBFE problems suggests many open questions, including creating approximation algorithms for other classes of Boolean functions, proving hardness results, and determining adaptivity gaps.

In the remainder of this section, we provide additional information about our results and about the relation between the problems that we consider. We defer detailed definitions of the relevant problems to Section 3.

Bounds on ADG and the adaptive greedy algorithm. Our new algorithm for the SSSC problem-ADG-is an extension of the dual greedy algorithm for SSC due to Fujito, which is a generalization of Hochbaum's primal-dual algorithm for the classical set cover problem [Fujito 1999, 2000]. We prove that it achieves an approximation factor of $\alpha$, where $\alpha$ is a ratio that depends on the cover constructed by the algorithm.

Golovin and Krause showed that the adaptive greedy algorithm achieves a $(\ln Q+1)$ approximation, where $Q$ is the goal utility. We prove that the algorithm achieves a $2(\ln P+1)$-approximation in the binary case, where $P$ is the maximum utility that can be contributed by a single item. (The bound is $k(\ln P+1)$ in the $k$-ary case.) Our bound generalizes Wolsey's bound for (nonadaptive) SSC [Wolsey 1982], except for the additional factor of 2 . Wolsey's bound generalized the $(\ln s+1)$ bound for standard set cover, where $s$ is the maximum size of one of the input subsets (see Fujito [2000]).

The preceding approximation bounds for the adaptive greedy algorithm and ADG compare the expected cost of the cover computed by the approximation algorithm to the expected cost incurred by an optimal strategy. We give additional bounds that are in terms of the expected cost of the optimal cover, which is a lower bound on the expected cost incurred by an optimal strategy.

Results on SBFE. In the SBFE problem, the task is to determine the value of a given Boolean function $f$ on an unknown input $x$, when each bit $x_{i}$ of $x$ can only be determined by performing a test that has $\operatorname{cost} c_{i}$. For each $x_{i}$, we are given the probability $p_{i}$ that $x_{i}=1$. The $x_{i}$ are independent. The problem is to determine the sequential, adaptive order in which to test the bits so as to minimize expected cost.

SBFE problems arise in diverse application areas. For example, in medical diagnosis, the $x_{i}$ might correspond to medical tests, and $f(x)=1$ if the patient has a particular disease. In database query optimization, $f$ could correspond to a Boolean query, on predicates corresponding to $x_{1}, \ldots, x_{n}$, that has to be evaluated on every tuple in the database [Ibaraki and Kameda 1984; Krishnamurthy et al. 1986; Deshpande and Hellerstein 2008; Srivastava et al. 2006]. In operations research, the SBFE problem is known as sequential testing of Boolean functions [Ünlüyurt 2004]. In learning theory, the SBFE problem has been studied in the context of learning with attribute costs [Kaplan et al. 2005]. There have been previous works on exact algorithms for SBFE problems, but there is little previous work on approximation algorithms [Ünlüyurt 2004; Kaplan et al. 2005].

If $f$ is given by its truth table, the SBFE problem can be exactly solved in time polynomial in the size of the truth table using dynamic programming, as in Guijarro et al. [1999] and Nijssen and Fromont [2007]. The following algorithm solves the SBFE problem with an approximation factor of $n$ for any function $f$, even under arbitrary distributions: test the variables in increasing cost order. This strategy was proven in 
Kaplan et al. [2005] to give a factor of $n$ approximation in the worst-case cost setting, and their proof holds for the expected-cost setting as well. We thus consider a factor of $n$ approximation to be trivial.

Our approach to solving SBFE problems is to reduce them to instances of the SSSC problem. The 3-approximation algorithm that we present for evaluating LTFs is obtained by using this approach and applying ADG.

Results on stochastic min-knapsack. The SBFE problem for evaluating LTFs is closely related to the stochastic min-knapsack problem. We define the stochastic min-knapsack problem as the problem of adaptively filling an initially empty knapsack with items from a given set of $n$ items until the total size of the items in the knapsack is at least a fixed threshold $\theta$ or all items have been used. The size of each item is randomly chosen from a finite set of possible item sizes according to a known distribution. The size of an item is not revealed until it is placed in the knapsack. Each item has a nonnegative cost, and the sizes of different items are independent. The goal is to minimize the expected total cost of the items placed in the knapsack.

There are several previous results on the stochastic (max) knapsack problem and on (deterministic) min-knapsack (e.g., see Carr et al. [2000], Dean et al. [2004], Bhalgat et al. [2011], and Bhalgat [2011]). Although min-knapsack and max-knapsack problems are equivalent in the exact case, the corresponding approximation problems are not equivalent. Derman et al. [1978] gave results on stochastic versions of the minknapsack problem when item sizes are exponentially distributed.

A minor modification of our algorithm for linear threshold evaluation yields a 3approximation algorithm for stochastic min-knapsack. We also get a 2-approximation algorithm for the variant of stochastic min-knapsack in which the distributions on the sizes are such that the threshold $\theta$ can always be reached or exceeded. These are the first constant-factor approximations for stochastic min-knapsack. An $O(\log \theta)$ approximation algorithm, for the general case, follows from work of Im et al. on the stochastic submodular ranking problem [Im et al. 2012; Im and Nagarajan 2011] when the item sizes and threshold $\theta$ are nonnegative integers.

If our algorithm for stochastic min-knapsack is applied to the much simpler deterministic version of the problem (the min-knapsack problem), our algorithm becomes the same as the algorithm of Carnes and Shmoys [2008]. However, they did not frame the problem as a special case of SSC.

Limitations of the Q-value approach. Before presenting our results on evaluating LTFs using ADG, we consider solving SBFE problems using an approach that exploits existing techniques. In this approach, we reduce the SBFE problem to an SSSC problem, solve the SSSC problem using the adaptive greedy algorithm, and apply the bound of Golovin and Krause [2011] for that algorithm. We formalize this approach and call it the $Q$-value approach.

We show that the $Q$-value approach easily yields an $O(\log k d)$-approximation algorithm for evaluating CDNF formulas (or decision trees), where $k$ is the number of terms in the DNF and $d$ is the number of clauses in the conjunctive normal form $(\mathrm{CNF})$. Previously, Kaplan et al. [2005] gave an algorithm also achieving an $O(\log k d)$ approximation (even with respect to the expected certificate cost), but only for monotone CDNF formulas, unit costs, and for the uniform distribution [Kaplan et al. 2005].

We then show that the $Q$-value approach provably cannot yield a sublinear approximation result for evaluating LTFs. Thus, it cannot be used to obtain the 3approximation algorithm obtained in this article by using ADG. We also prove that the $Q$-value approach cannot yield a sublinear approximation result for evaluating readonce DNF formulas, or DNF formulas with two literals per term, even though there are poly-time exact algorithms for those problems [Kaplan et al. 2005; Greiner et al. 2002]. 
Additional applications of the Q-value approach. At the end of this article, we include some additional applications of variants of the $Q$-value approach, using bounds on the adaptive greedy algorithm and ADG. We give two algorithms with approximation factors of $O\left(\log m D_{\text {avg }}\right)$ and $D_{\max }$, respectively, solving the problem of simultaneous evaluation of $m$ LTFs with integer coefficients. Here, $D_{\text {avg }}$ and $D_{\max }$ are the average and maximum, over the $m$ formulas, of the sum of the magnitude of the coefficients. These results generalize results of Liu et al. [2008] for shared filter ordering. We also improve one of the results of Liu et al. for that problem.

We give an $O\left(\log \left(m D_{\max }\right)\right.$ )-approximation algorithm for ranking a set of $m$ linear functions $a_{1} x_{1}+\cdots+a_{n} x_{n}$ (not linear threshold functions), defined over $\{0,1\}^{n}$, by their output values, in our stochastic setting. This problem arises in Web search and database query processing. For example, we might need to rank a set of documents or tuples by their "scores," where the linear functions compute the scores over a set of unknown properties such as user preferences or data source reputations.

\section{STRUCTURE OF THE ARTICLE}

We begin with a discussion of related work in Section 3. Section 4 introduces necessary background and notation and contains the formal definitions of the SSSC, SBFE, and min-knapsack problems. Section 5 contains a discussion of the connection between the SSSC problem and the SBFE problem, and presents positive and negative results for SBFE problems using the $Q$-value approach. Section 6 introduces and analyzes ADG. In Section 7, we use ADG to obtain approximation algorithms for linear threshold evaluation and for stochastic min-knapsack. Section 8 contains our new bound for the adaptive greedy algorithm. In Section 9, we give approximation bounds for ADG and the adaptive greedy algorithm in terms of the optimal expected cover cost. Finally, in Section 10, we present additional results on ranking and simultaneous function evaluation using variants of the $Q$-value approach.

\section{RELATED WORK}

There is a well-known algorithm that exactly solves the SBFE problem for disjunctions: test the $x_{i}$ in increasing order of ratio $c_{i} / p_{i}$ (e.g., see Garey [1973]). A symmetric algorithm works for conjunctions. There is also a poly-time exact algorithm for evaluating a $k$-of- $n$ function (i.e., a function that evaluates to 1 if and only if at least $k$ of the $x_{i}$ are equal to 1) [Salloum 1979; Ben-Dov 1981; Salloum and Breuer 1984; Chang et al. 1990]. There is a poly-time exact algorithm for evaluating a read-once DNF formula $f$ (a DNF formula in which every literal appears at most once), but the complexity of the problem is open when $f$ is a general read-once formula [Boros and Ünlüyurt 2000; Greiner et al. 2002, 2006].

The SBFE problem is NP-hard for LTFs, even with unit costs [Cox et al. 1989]. For the special case of unit costs and the uniform distribution, testing the variables in decreasing order of the magnitude of their coefficients is optimal [Boros and Ünlüyurt 1999; Fiat and Pechyony 2004], and the special case of unit coefficients is solvable in polynomial time because it is the same as the problem of evaluating $k$-of- $n$ functions.

Allen et al. [2013] gave results for the SBFE problem on classes of monotone DNF formulas. A survey by Ünlüyurt [2004] covers other results on exactly solving SBFE problems.

There is a sample version of the evaluation problem, where the input is a sample of size $m$ of $f$ (i.e., a set of $m$ pairs $(x, f(x))$ ), and the problem is to build a decision tree in polynomial time that computes $f$ correctly on the $x$ in the sample and minimizes the average cost of evaluation over the sample. Golovin et al. [2010] and Bellala et al. [2012] developed $O(\log m)$-approximation algorithms for this problem for arbitrary $f$, and there is a 4-approximation algorithm when $f$ is a conjunction [Bar-Noy et al. 
1998; Feige et al. 2002; Munagala et al. 2005]. Cicalese et al. [2013] subsequently gave an algorithm achieving the same approximation factor when there is a probability distribution on the elements in the sample, and the goal is to minimize the expected cost with respect to that distribution. Moshkov and Chikalov [1997] proved a related bound in terms of a combinatorial measure of the sample. Moshkov [2003] gave an $O(\log m)$-algorithm for a worst-case cost variant of this problem.

The work of Im et al. on stochastic submodular ranking has applications to the problem of simultaneous evaluation of Boolean functions [Im et al. 2012; Im and Nagarajan 2011]. The goal in that problem is to minimize cost, which in their setting corresponds to minimizing the maximum of the cover times for the functions. Im et al. focus on the problem of minimizing the sum of the cover times.

Several nonadaptive versions of standard and submodular set covers have been studied. For example, Iwata and Nagano [2009] studied the "submodular cost set cover" problem, where the cost of the cover is a submodular function that depends on which subsets are in the cover. Beraldi and Ruszczynski [2002] addressed a set cover problem where the set of elements covered by each input subset is a random variable, and full coverage must be achieved with a certain probability.

Prior to the work of Golovin et al., Golovin and Krause [2011] and Liu et al. [2008] analyzed the adaptive greedy algorithm for a restricted version of the stochastic set cover problem studied by Goemans and Vondrák, described in Section 1. The analysis of Liu et al. does not extend to the SSSC problem. To apply the adaptive greedy algorithm to threshold evaluation and min-knapsack, we need the generalization to the submodular case.

Kaplan et al. [2005] also considered the problem of minimizing the expected cost of evaluating a Boolean function $f$ with respect to a given arbitrary probability distribution, where the distribution is given by a conditional probability oracle. In the work of Kaplan et al., the goal of evaluation differs slightly from ours in that they require the evaluation strategy to output an explanation of the function value upon termination. They give as an example the case of evaluating a DNF that is identically true; they require testing of the variables in one term of the DNF to output that term as a certificate. In contrast, under our definitions, the optimal strategy for evaluating an identically true DNF formula is a zero-cost strategy that simply outputs true and performs no tests.

Charikar et al. [2002] considered the problem of minimizing the worst-case ratio between the cost of evaluating $f$ on an input $x$ and the minimum cost of a certificate contained in $x$. There are also works on building identification trees of minimum average cost, given $S \subseteq\{0,1\}^{n}$, but that problem is fundamentally different than function evaluation because each $x \in S$ must have its own leaf (see Adler and Heeringa [2012]).

There are several previous combinatorial and noncombinatorial 2-approximation algorithms for (deterministic) min-knapsack (see Han and Makino [2010]), and this problem also has a PTAS [Carr et al. 2000]. Han and Makino [2010] considered an online version of min-knapsack where the items are given one-by-one over time.

There are several constant-factor approximation algorithms for stochastic ( $\max )$ knapsack problems, including a $(2+\epsilon)$-approximation [Dean et al. 2004; Bhalgat et al. 2011; Bhalgat 2011].

\section{PRELIMINARIES}

A table with the main notation used in this article is provided in Appendix A.

Basic notation and definitions. Let $\mathcal{O}=\{0,1, \ldots, k-1\}$ for some integer $k \geq 2$. In the binary case, $k=2$. 
A partial assignment is a vector $b \in(\mathcal{O} \cup\{*\})^{n}$. We view $b$ as an assignment to variables $x_{1}, \ldots, x_{n}$. We will use $b \in(\mathcal{O} \cup\{*\})^{n}$ to represent the outcomes of $k$-ary tests, where for $l \in \mathcal{O}, b_{i}=l$ indicates that test $i$ has outcome $l$, and $b_{i}=*$ indicates that the outcome of test $i$ is not known.

For partial assignments $a, b \in(\mathcal{O} \cup\{*\})^{n}, a$ is an extension of $b$, written $a \succeq b$, if $a_{i}=b_{i}$ for all $b_{i} \neq *$. We also say that $b$ is contained in $a$.

Let $N=\{1, \ldots, n\}$. In what follows, we assume that utility functions are integer valued. In the context of standard work on submodularity, a utility function is a set function $g: 2^{N} \rightarrow \mathbb{Z}_{\geq 0}$. Given $S \subseteq N$ and $j \in N, g_{S}(j)$ denotes the quantity $g(S \cup\{j\})-g(S)$.

We will also use the term utility function to refer to a function $g:(\mathcal{O} \cup\{*\})^{n} \rightarrow \mathbb{Z}_{\geq 0}$ defined on partial assignments. Let $g:(\mathcal{O} \cup\{*\})^{n} \rightarrow \mathbb{Z}_{\geq 0}$ be such a utility function, and let $b \in(\mathcal{O} \cup\{*\})^{n}$. We define $g(S, b)=g\left(b^{\prime}\right)$, where $b^{\prime}$ is the partial assignment such that $b_{i}^{\prime}=b_{i}$ for $i \in S$ and $b_{i}^{\prime}=*$ otherwise. For $j \in N$, we define $g_{S, b}(j)=g(S \cup\{j\}, b)-g(S, b)$. If $j \notin S$ and $b_{j} \neq *$, this denotes the increase in utility resulting from adding $j$ to $S$ when $x_{j}$ is assigned the value indicated by $b_{j}$.

For $l \in \mathcal{O} \cup\{*\}$, we define $b_{x_{i} \leftarrow l}$ to be the partial assignment that is identical to $b$ except that $b_{i}=l$. We define $g_{b}(i, l)=g\left(b_{x_{i} \leftarrow l}\right)-g(b)$ if $b_{i}=*$ and $g_{b}(i, l)=0$ otherwise. When $b$ represents test outcomes and test $i$ has not been performed yet, $g_{b}(i, l)$ is the change in utility that would result from adding test $i$ with outcome $l$.

For $p=\left(p_{1}, \ldots, p_{n}\right)$ where $0 \leq p_{i} \leq 1$, we use $D_{p}$ to denote the product distribution on $\{0,1\}^{n}$ where $p_{i}=\operatorname{Prob}\left[x_{i}=1\right]$. For $b \in\{0,1, *\}^{n}, p(b)$ is the probability that a random $x$ drawn from $D_{p}$ will have the same values as $b$ for all $j$ such that $b_{j} \neq *$. In other words, $p(b)=\left(\prod_{j: b_{j}=1} p_{j}\right)\left(\prod_{j: b_{j}=0}\left(1-p_{j}\right)\right)$.

Given an distribution $D$ on $\mathcal{O}^{n}, a \sim D$ denotes that $a$ is a random element drawn from distribution $D$. For partial assignment $b \in(\mathcal{O} \bigcup\{*\})^{n}, i \in N$, and fixed product distribution $D$ on $\mathcal{O}^{n}, E\left[g_{b}(i)\right]$ denotes the expected value of $g_{b}\left(i, a_{i}\right)$, where the expectation is with respect to $a \sim D$, conditioned on $a \succeq b$. If $b_{i} \neq *, E\left[g_{b}(i)\right]=0$. In the testing context, when $b$ represents the outcomes of tests performed so far, then if $b_{i}=*$, $E\left[g_{b}(i)\right]$ is the expected increase in utility that would be obtained by testing $i$ next. In the binary case, where $D=D_{p}, E\left[g_{b}(i)\right]=p_{i} g_{b}(i, 1)+\left(1-p_{i}\right) g_{b}(i, 0)$.

Utility function $g:(\mathcal{O} \cup\{*\})^{n} \rightarrow \mathbb{Z}_{\geq 0}$ is monotone if for $b \in(\mathcal{O} \cup\{*\})^{n}, i \in N$ such that $b_{i}=*$, and $l \in(\mathcal{O} \cup\{*\}), g\left(b_{x_{i} \leftarrow l}\right)-g(b) \geq 0$; in other words, additional information can only increase utility. Utility function $g$ is submodular if for all $b, b^{\prime} \in(\mathcal{O} \cup\{*\})^{n}$ and $l \in \mathcal{O}, g\left(b_{x_{i} \leftarrow l}\right)-g(b) \geq g\left(b_{x_{i} \leftarrow l}^{\prime}\right)-g\left(b^{\prime}\right)$ whenever $b^{\prime} \succeq b$ and $b_{i}=b_{i}^{\prime}=*$. In the testing context, if the $n$ test outcomes are predetermined, submodularity means that the value of a given test (measured by the increase in utility) will not increase if we delay that test until later.

Given Boolean function $f:\{0,1\}^{n} \rightarrow\{0,1\}$, a partial assignment $b \in\{0,1, *\}^{n}$ is a 0 -certificate (1-certificate) of $f$ if $f(a)=0(f(a)=1)$ for all $a$ such that $a \succeq b$. Given a cost vector $c=\left(c_{1}, \ldots, c_{n}\right)$, the cost of a certificate $b$ is $\sum_{j: b_{j} \neq *} c_{j}$.

A literal is a Boolean variable or its negation. A term is a possibly empty conjunction $(\wedge)$ of literals. If the term is empty, all assignments satisfy it. A clause is a possibly empty disjunction $(V)$ of literals. If the clause is empty, no assignment satisfies it. A DNF formula is either the constant 0 , the constant 1 , or a formula of the form $t_{1} \vee \cdots \vee t_{k}$, where $k \geq 1$ and each $t_{i}$ is a term. Likewise, a CNF formula is either the constant 0 , the constant 1 , or a formula of the form $c_{1} \wedge \cdots \wedge c_{k}$, where each $c_{i}$ is a clause. The size of a $\mathrm{DNF}$ (CNF) formula is the number of terms (clauses) it contains; if it is the constant 0 or 1 , its size is 1 . A DNF formula is monotone if it contains no negations. A read-once DNF formula is a DNF formula in which each variable appears at most once.

An LTF with integer coefficients has the form $\sum_{i=1}^{n} a_{i} x_{i} \geq \theta$, where the $a_{i}$ and $\theta$ are integers. It represents the function $f:\{0,1\}^{n} \stackrel{\rightarrow=1}{\rightarrow}\{0,1\}$ such that $f(x)=1$ if 
$\sum_{i=1}^{n} a_{i} x_{i} \geq \theta$ and $f(x)=0$ otherwise. LTFs generalize OR formulas (in which all of the $a_{i}$ and $\theta$ are set to 1 ) and $k$-of- $n$ functions (in which all $a_{i}=1$ and $\theta=k$ ).

The SSSC problem. Let $\mathcal{O}=\{0,1, \ldots, k-1\}$ be a finite set of $k$ states, where $k \geq 2$. We call $\mathcal{O}$ the state space. To simplify the exposition, we define the SSSC problem in terms of integer-valued utility functions.

We will focus our attention on the binary SSSC problem, where $k=2$. We will briefly discuss extensions to the $k$-ary version of the problem for $k>2$.

The input to the SSSC problem consists of a set $N=\{1, \ldots, n\}$ of items, a cost vector $\left(c_{1}, \ldots, c_{n}\right)$, where each $c_{j} \geq 0$, a probability vector $p=\left(p_{1}, \ldots, p_{n}\right)$ where $0 \leq p_{i} \leq 1$, an integer $Q \geq 0$, and an oracle for a utility function $g:(\mathcal{O} \cup\{*\})^{n} \rightarrow \mathbb{Z}_{\geq 0}$. Further, utility function $g(x)$ satisfies the following property: there is an integer $Q \geq 0$ such that $g(x)=Q$ if $x \in \mathcal{O}^{n}$. We call $Q$ the goal utility. We say that $b \in(\mathcal{O} \cup\{*\})^{n}$ is a cover if $g(b)=Q$. The cost of cover $b$ is $\sum_{j: b_{j} \neq *} c_{j}$.

An algorithm for the SSSC problem must sequentially choose items from $N$. Each item $j \in N$ has a state $x_{j}$, where $x_{j} \in \mathcal{O}$. The $x_{j}$ are random variables. The vector $x=\left(x_{1}, \ldots, x_{n}\right)$, corresponding to the states of the $n$ items, is distributed according to the product distribution $D_{p}$. The states of the $n$ items are initially unknown. Each time the algorithm chooses an item, it "tests" the item, incurring cost $c_{j}$. When an item is tested, the state of $x_{j}$ is revealed. The states of the items tested so far are represented by a partial assignment $b \in(\mathcal{O} \cup *)^{n}$. The algorithm must choose items until $g(b)=Q-$ that is, until $b$ is a cover. The choice of items can be adaptive, in that the choice of the next item can depend on the states of previous items.

We say that the algorithm computes a strategy for choosing the items. The strategy corresponds to a decision tree, indicating the adaptive order in which items should be chosen. The algorithm and its computed strategy are optimal if the algorithm minimizes the expected cost incurred on a random state vector $x$ drawn from $D_{p}$.

The running time of the algorithm is the maximum, over all $x$, of the time taken to achieve a cover. We assume that each test takes constant time and that each call to the oracle for a monotone, submodular utility function $g$ takes constant time. The running time includes the time taken to repeatedly determine the next test to perform.

The SBFE problem. The input to the SBFE problem is a representation of a Boolean function $f\left(x_{1}, \ldots, x_{n}\right)$ from a fixed class of representations $C$; a probability vector $p=$ $\left(p_{1}, \ldots, p_{n}\right)$, where $0<p_{i}<1$; and a real-valued cost vector $\left(c_{1}, \ldots, c_{n}\right)$, where $c_{i} \geq 0$. An algorithm for this problem must compute and output the value of $f$ on an $x \in$ $\{0,1\}^{n}$, drawn randomly from the product distribution $D_{p}$ defined by $\left(p_{1}, \ldots, p_{n}\right)$, where $p_{i}=\operatorname{Prob}\left[x_{i}=1\right]$. However, the algorithm is not given access to $x$. Instead, it can discover the value of any $x_{i}$ by "testing" it, at a cost of $c_{i}$. The algorithm must perform the tests sequentially, each time choosing the next test to perform. The algorithm can be adaptive, so the choice of the next test can depend on the outcomes of the previous tests.

The expected cost of the algorithm is the cost it incurs on a random $x$ from $D_{p}$. (Since each $p_{i}$ is strictly between 0 and 1 , the algorithm must continue doing tests until it has obtained a 0-certificate or 1-certificate for the function.) The algorithm is optimal if it has minimum expected cost with respect to $D_{p}$.

The algorithm corresponds to a Boolean decision tree (testing strategy) computing $f$. The running time of the algorithm is the maximum, over all inputs $x$, of the time it takes to evaluate $f$ on $x$, assuming that each test takes constant time. This running time includes the time required to repeatedly determine the next test to perform.

The stochastic min-knapsack problem. The input to the stochastic min-knapsack problem is a set $N=\{1, \ldots, n\}$ of items; a nonnegative threshold value $\theta \geq 0$; and a 
real-valued cost vector $\left(c_{1}, \ldots, c_{n}\right)$, where $c_{i} \geq 0$, along with a description, for each item $i$, of a probability distribution on the size $s_{i} \geq 0$ of item $i$. We assume in this work that each $s_{i}$ has only a finite number of possible values. We expect our results to extend to continuous distributions for $s_{i}$ but defer that to future work.

We assume that the sizes of the items are independent. An algorithm for this problem incrementally chooses items to place in a subset $S$, starting from $S=\emptyset$, until either $\sum_{j \in S} s_{i} \geq \theta$ or $S$ contains all $n$ items. The choice of the next item can depend on the sizes of the previous items chosen. The size $s_{i}$ of the $i$ th item is not revealed until the item is chosen for inclusion in $S$, and once an item is added to $S$, it cannot be removed. The goal is to minimize the expected value of $\sum_{j \in S} c_{i}$.

The adaptive greedy algorithm for SSSC. The classical set cover problem has a simple greedy approximation algorithm that chooses the subset with the "best bang for the buck"-that is, the subset covering the most new elements per unit cost. The generalization of this algorithm to the SSC problem, studied by Wolsey [1982], chooses the element that adds the maximum additional utility per unit cost. The adaptive greedy algorithm of Golovin and Krause, for the SSSC problem, is a further generalization. It chooses the element with the maximum expected increase in utility per unit cost. (Golovin and Krause actually formulated the adaptive greedy algorithm for use in solving a somewhat more general problem than SSSC.)

Golovin and Krause proved that the adaptive greedy algorithm is a $(\ln Q+1)$ approximation algorithm for SSSC, where $Q$ is the goal utility. We will make repeated use of this bound.

\section{FUNCTION EVALUATION AND THE SSSC PROBLEM}

Before presenting our new results on the SSSC problem, we show how to use existing results on the SSSC problem to obtain results for SBFE problems.

\subsection{The $Q$-Value Approach and CDNF Evaluation}

Let $f\left(x_{1}, \ldots, x_{n}\right)$ be a Boolean function. Let $g:\{0,1, *\}^{n} \rightarrow \mathbb{Z}_{\geq 0}$ be a utility function. We say that $g$ is assignment feasible for $f$, with goal value $Q$, if (1) $g$ is monotone and submodular, (2) $g(*, *, \ldots, *)=0$, and (3) for $b \in\{0,1, *\}^{n}, g(b)=Q$ if and only if $b$ is a 0 -certificate or a 1-certificate of $f$.

We consider the following approach to solving SBFE problems, which we call the $Q$-value approach. To evaluate $f$, we construct an assignment feasible utility function $g$ for $f$ with goal value $Q$. We then run the adaptive greedy algorithm on the resulting SSSC problem. Because $g(b)=Q$ if and only if $b$ is either a 0 -certificate or a 1 -certificate of $f$, the strategy computed by the adaptive greedy algorithm is a solution to the SBFE problem for $f$. By the bound on the adaptive greedy algorithm, this strategy is within a factor of $(\ln Q+1)$ of optimal. (In Section 9 , we show that under the uniform distribution, the solution is actually within a factor of $O(\ln Q)$ of the expected cost of the optimal certificate.)

The challenge in using the preceding approach is in constructing $g$. Not only must $g$ be assignment feasible but $Q$ should be subexponential to obtain a good approximation bound. We will use the following lemma in our construction of $g$. This lemma has appeared in several previous works, either implicitly or explicitly [Guillory and Bilmes 2011; Bellala et al. 2012; Golovin et al. 2010].

Lemma 1. Let $g_{0}:\{0,1, *\}^{n} \rightarrow \mathbb{Z}_{\geq 0}, g_{1}:\{0,1, *\}^{n} \rightarrow \mathbb{Z}_{\geq 0}$, and $Q_{0}, Q_{1} \in \mathbb{Z}_{\geq 0}$ be such that $g_{0}$ and $g_{1}$ are monotone, submodular utility functions, $g_{0}(*, *, \ldots, *)=g_{1}(*, *, \ldots, *)=$ 0 , and $g_{0}(a) \leq Q_{0}$ and $g_{1}(a) \leq Q_{1}$ for all $a \in\{0,1\}^{n}$. 
Let $Q_{\vee}=Q_{0} Q_{1}$, and let $g_{\vee}:\{0,1, *\}^{n} \rightarrow \mathbb{Z}_{\geq 0}$ be such that $g_{\vee}(b)=Q_{\vee}-\left(Q_{0}-g_{0}(b)\right)\left(Q_{1}-\right.$ $\left.g_{1}(b)\right)$.

Let $Q_{\wedge}=Q_{0}+Q_{1}$, and let $g_{\wedge}:\{0,1, *\}^{n} \rightarrow \mathbb{Z}_{>0}$ be such that $g_{\wedge}(b)=g_{0}(b)+g_{1}(b)$.

Then $g_{\vee}$ and $g_{\wedge}$ are monotone and submodular, and $g_{\vee}(*, \ldots, *)=g_{\wedge}(*, \ldots, *)=0$. For $b \in\{0,1, *\}^{n}, g_{\vee}(b)=Q_{\vee}$ if and only if $g_{0}(b)=Q_{0}$ or $g_{1}(b)=Q_{1}$, or both. Further, $g_{\wedge}(b)=Q_{\wedge}$ if and only if $g_{0}(b)=Q_{0}$ and $g_{1}(b)=Q_{1}$.

Using the $Q$-value approach, it is easy to obtain an algorithm for evaluating CDNF formulas. A CDNF formula for Boolean function $f$ is a pair $\left(\phi_{0}, \phi_{1}\right)$, where $\phi_{0}$ and $\phi_{1}$ are $\mathrm{CNF}$ and DNF formulas for $f$, respectively.

THEOREM 1. There is a polynomial-time $O(\log k d)$-approximation algorithm solving the SBFE problem for CDNF formulas, where $k$ is the number of clauses in the CNF and $d$ is the number of terms in the DNF.

PRoof. Let $\phi_{0}$ be the CNF and $\phi_{1}$ be the DNF. Let $f$ be the Boolean function defined by these formulas. Let $k$ and $d$ be the number of clauses and terms of $\phi_{0}$ and $\phi_{1}$, respectively. Let $g_{0}:\{0,1, *\}^{n} \rightarrow \mathbb{Z}_{\geq 0}$ be such that for $a \in\{0,1, *\}^{n}, g_{0}(a)$ is the number of terms of $\phi_{1}$ set to 0 by $a$ (i.e., terms with a literal $x_{i}$ such that $a_{i}=0$, or a literal $\neg x_{i}$ such that $\left.a_{i}=1\right)$. Similarly, let $g_{1}(a)$ be the number of clauses of $\phi_{0}$ set to 1 by $a$. Clearly, $g_{0}$ and $g_{1}$ are monotone and submodular. Partial assignment $b$ is a 0 -certificate of $f$ if and only if $g_{0}(b)=d$ and a 1-certificate of $f$ if and only if $g_{1}(b)=k$. Applying the disjunctive construction of Lemma 1 to $g_{1}$ and $g_{0}$ yields a utility function $g$ that is assignment feasible for $f$ with goal value $Q=k d$. Applying the adaptive greedy algorithm and its $(\ln Q+1)$ bound yields the theorem.

Given a decision tree for a Boolean function $f$, a CNF (DNF) for $f$ can be easily computed using the paths to the 0-leaves (1-leaves) of the tree. Thus, the preceding theorem gives an $O(\ln t)$-approximation algorithm for evaluating decision trees, where $t$ is the number of leaves.

Having provided a formal proof of the preceding theorem, we now provide an intuitive explanation of the construction used in it. Let $\tilde{T}$ be the set of terms in a DNF formula for $f$, and let $\tilde{C}$ be the set of clauses in a CNF formula for $f$. We can view each pair $(T, C)$, where $T \in \tilde{T}$ and $C \in \tilde{C}$ as a conflicting pair, in that it is not possible for an assignment $a$ to simultaneously satisfy $T$ and falsify $C$.

Given a partial assignment $b$, define $T(b)$ to be the set of terms of $\tilde{T}$ that are not falsified by the variables assigned values by $b$, and define $C(b)$ to be the set of clauses of $\tilde{C}$ that are not satisfied by the variables assigned values by $b$. Thus, $T(b)$ is the set of terms that can be satisfied by some extension of $b$, and $C(b)$ is the set of clauses that can be falsified by some extension of $b$. If there is a full extension $a$ of $b$ such that $f(a)=1$, and if there is a full extension $a^{\prime}$ of $b$ such that $f(a)=0$, then $T(b)$ contains at least one term (namely, a term satisfied by $a$ ), and $C(b)$ contains at least one clause (namely a clause falsified by $a^{\prime}$ ). Conversely, if $f$ has the same value on all extensions of $b$, then either $T(b)$ or $C(b)$ is empty. It follows that discovering the value of $f$ on hidden assignment $a$ is equivalent to gathering enough information so that the corresponding partial assignment $b$ is such that there is no conflicting pair $T, C$ such that $T \in T(b)$ and $C \in C(b)$.

Then we can rephrase our preceding definitions: $Q_{1}=|\tilde{C}|, Q_{0}=|\tilde{T}|$ and $Q=|\tilde{T}| \cdot|\tilde{C}|$. Then $g_{1}(b)=|\tilde{C}|-C(b)$ and $g_{0}(b)=|\tilde{T}|-T(b)$. Hence, $g(b)$ is the number of $(T, C)$ conflicting pairs eliminated by the tests whose values are given by $b$. This construction reduces the SBFE problem on CDNF to a stochastic set cover problem, where the goal is to cover the conflicting pairs with variables. 


\subsection{Limitations of the $Q$-Value Approach}

The $Q$-value approach depends on finding an assignment feasible utility function $g$ for $f$. We first demonstrate that such a generic $g$ exists for all Boolean functions $f$. For $i \in\{0,1\}$, let $Q_{i}=\left|\left\{a \in\{0,1\}^{n} \mid f(a)=i\right\}\right|$. For partial assignment $b$, let $g_{i}(b)=$ $Q_{i}-\left|\left\{a \in\{0,1\}^{n} \mid a \succeq b, f(a)=i\right\}\right|$ with goal value $Q_{i}$. Then $g_{0}, Q_{0}, g_{1}$, and $Q_{1}$ obey the properties of Lemma 1. Apply the disjunctive construction in that lemma, and let $g$ be the resulting utility function. Then $g$ is assignment feasible for $f$ with goal value $Q=Q_{1} Q_{0}$. In fact, this $g$ is precisely the utility function that would be constructed by the approximation algorithm of Golovin et al. [2010] for computing a consistent decision tree of min-expected cost with respect to a sample, if we take the sample to be the set of all $2^{n}$ entries $(x, f(x))$ in the truth table of $f$. The goal value $Q$ of this $g$ is $2^{\theta(n)}$, so in this case the bound for the adaptive greedy algorithm, $(\ln Q+1)$, is linear in $n$.

Since we want a sublinear approximation factor, we would instead like to construct an assignment-feasible utility function for $f$ whose $Q$ is subexponential in $n$. However, we now show that this is impossible even for some simple Boolean functions $f$. We begin by introducing the following combinatorial measure of a Boolean function, which we call its $Q$-value.

$Q$-value. We define the $Q$-value of a Boolean function $f:\{0,1\}^{n} \rightarrow\{0,1\}$ to be the minimum integer $Q$ such that there exists an assignment feasible utility function $g$ for $f$ with goal value $Q$.

The preceding generic $g$ shows that the $Q$-value of every $n$-variable Boolean function is at most $2^{O(n)}$.

We now prove lower bounds on $Q$ for some particular functions. First, consider the Boolean function $f:\{0,1\}^{n} \rightarrow\{0,1\}$, where $n$ is even, represented by the read-once DNF formula $\phi=t_{1} \vee t_{2} \vee \cdots \vee t_{n / 2}$, where each $t_{i}=x_{i} x_{n / 2+i}$. Suppose that $g$ is an assignmentfeasible utility function for $f$. This function has the following simple property: if you set one variable in each of the first $m$ terms of $\phi$ to be 1 , where $1 \leq m \leq n / 2$, this is not sufficient to force the value of the function to be 1 . However, if you then set the remaining variable in the $m$ th term to 1 , this does force the function value to 1 . The following technical lemma applies to this particular function $f$ and to other functions that satisfy a version of this property.

Lemma 2. Let $f\left(x_{1}, \ldots, x_{n}\right)$ be a Boolean function, where $n$ is even. Further, let $f$ be such that for all $n^{\prime} \leq n / 2$, and for all $b \in\{0,1, *\}^{n}$, if $b_{i}=b_{n / 2+i}=*$ for all $i \in\left\{n^{\prime}+1, \ldots, n / 2\right\}$, the following properties hold:

(1) if for all $i \in\left\{1, \ldots, n^{\prime}\right\}$, exactly one of $b_{i}$ and $b_{n / 2+i}$ is equal to * and the other is equal to 1 , then $b$ is not a 0 -certificate or a 1-certificate of $f$, and

(2) if for all $i \in\left\{1, \ldots, n^{\prime}-1\right\}$, exactly one of $b_{i}$ and $b_{n / 2+i}$ is equal to * and the other is equal to 1 , and $b_{n^{\prime}}=b_{n / 2+n^{\prime}}=1$, then $b$ contains a 1-certificate of $f$.

Then the $Q$-value of $f$ is at least $2^{n / 2}$.

Proof. Let $g$ be an assignment-feasible utility function for $f$. For $i \in\{1, \ldots, n / 2\}$, let $t_{i}$ denote the pair of variables $\left\{x_{i}, x_{n / 2+i}\right\}$.

Construct a partial assignment $b$ to the variables $x_{i}$ as follows. Initialize $b$ to be the partial assignment setting all $x_{i}$ to $*$. Since $g$ is assignment feasible, $g(b)=0$. Setting both variables in $t_{1}$ to 1 in $b$ would increase the value of $g(b)$ by $Q$, since by Property (2), $b$ would then contain a 1-certificate. By submodularity, for at least one of the variables in $t_{1}$, setting only that variable to 1 , by itself, must yield an increase in $g$ of at least $Q / 2$. Set this variable to 1 in $b$ so that now $g(b) \geq Q / 2$, and $Q-g(b) \leq Q / 2$. 
If we now set both variables in $t_{2}$ to 1 , this would again increase $g(b)$ to be $Q$, for a total increase in utility of $Q-g(b)$. Thus, by submodularity, for at least one of the variables in $t_{2}$, setting only that variable to 1 would increase the value of $g(b)$ by at least $(Q-g(b)) / 2$, resulting in a utility value of at least $Q / 2+Q / 4$. Set that variable to 1 in $b$ as well. Continue this procedure for $i=3, \ldots, n / 2$, each time setting one variable in $t_{i}$ to 1 , such that the increase in utility is at least $(Q-g(b)) / 2$ each time. The resulting $b$ then satisfies the inequality $g(b) \geq Q / 2+Q / 4+\cdots+Q /\left(2^{n / 2}\right)$.

By Property (1), $b$ does not contain a certificate for $f$, so $g(b)<Q$. Hence, $Q-g(b) \leq$ $Q /\left(2^{n / 2}\right)$, and since $g(b)$ is an integer, $Q-g(b) \geq 1$ and $Q \geq 2^{n / 2}$. We have thus shown that the $Q$-value of $f$ is at least $2^{n / 2}$.

Using the preceding lemma, we obtain an exponential lower bound for the $Q$-value of the function represented by the read-once DNF formula considered earlier.

Theorem 2. Let $n$ be even. Let $f:\{0,1\}^{n} \rightarrow\{0,1\}$ be the Boolean function represented by the read-once $D N F$ formula $\phi=t_{1} \vee t_{2} \vee \cdots \vee t_{n / 2}$, where each $t_{i}=x_{i} x_{n / 2+i}$. The Q-value of $f$ is at least $2^{n / 2}$.

The preceding theorem shows that the $Q$-value approach will not yield a good approximation bound for either read-once DNF formulas or for DNF formulas with terms of length 2.

In the next theorem, we show that there is a particular LTF whose $Q$-value is at least $2^{n / 2}$. It follows that the $Q$-value approach will not yield a good approximation bound for LTFs either.

We note that the function described in the next theorem has been studied before. As mentioned in Hastad [1994], there is a lower bound of essentially $2^{n / 2}$ on the size of the largest integer coefficients in any representation of the function as an LTF with integer coefficients.

THEOREM 3. Let $f\left(x_{1}, \ldots, x_{n}\right)$ be the function defined for even $n$, whose value is 1 if and only if the number represented in binary by bits $x_{1} \ldots x_{n / 2}$ is strictly less than the number represented in binary by bits $x_{n / 2+1} \ldots x_{n}$ and 0 otherwise. The Q-value of $f$ is at least $2^{\text {n/2 }}$.

Proof. We define a new function: $f^{\prime}\left(x_{1}, \ldots, x_{n}\right)=f\left(\neg x_{1}, \ldots, \neg x_{n / 2}, x_{n / 2+1}, \ldots, x_{n}\right)$. In other words, $f^{\prime}\left(x_{1}, \ldots, x_{n}\right)$ is computed by negating the assignments to the first $n / 2$ variables and then computing the value of $f$ on the resulting assignment. Function $f^{\prime}$ obeys the conditions of Lemma 3 and thus has a $Q$-value of at least $2^{n / 2}$. Then $f$ also has a $Q$-value of at least $2^{n / 2}$, because the $Q$-value is not changed by the negation of input variables.

Given the limitations of the $Q$-value approach, we can ask whether there are good alternatives. Our new bound on the adaptive greedy algorithm is $O(\log P)$, where $P$ is the maximum amount of utility gained by testing a single variable $x_{i}$, so we might hope to use a $P$-value in place of a $Q$-value. However, this does not help much: testing all $n$ variables yields utility $Q$, so testing one of them alone must yield utility at least $Q / n$, implying that $P \geq Q / n$. Another possibility might be to exploit the fact that Golovin and Krause's bounds on the adaptive greedy algorithm apply to a more general class of utility functions than the assignment feasible utility functions, but we do not pursue that possibility. Instead, we give a new algorithm for the SSSC problem.

\section{ADAPTIVE DUAL GREEDY}

We now present and analyze ADG, our new algorithm for the SSSC problem. We assume unless otherwise noted that the state space is binary-that is, $\mathcal{O}=\{0,1\}$. 


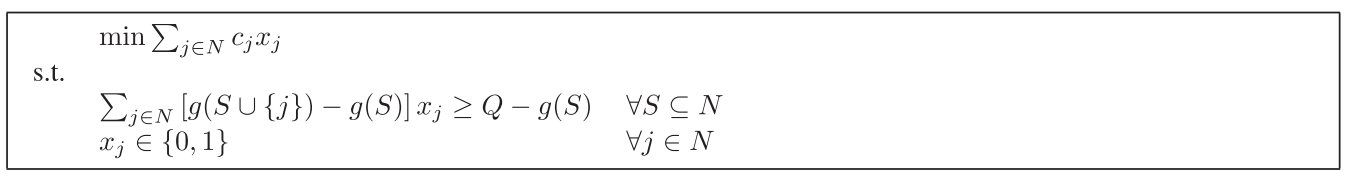

Fig. 1. Wolsey's IP for SSC.

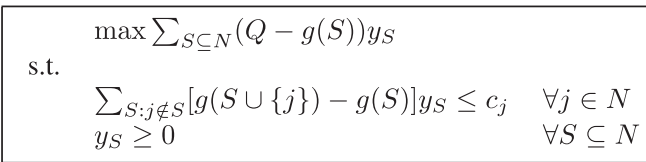

Fig. 2. Dual LP for SSC.

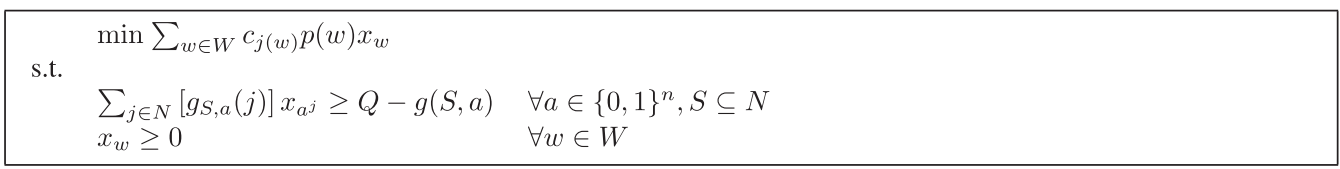

Fig. 3. LP1: the linear program for lower bounding ADG.

Like Fujito's dual greedy algorithm for the (deterministic) SSC problem, ADG is based on Wolsey's IP for that problem. We present Wolsey's IP in Figure 1. In the LP, function $g: 2^{N} \rightarrow \mathbb{Z}_{>0}$ is a set function that is monotone and submodular, and $Q=g(N)$.

The $n$ variables of the IP correspond to the set $N$ of elements that can be used to form the cover. There are $2^{n}$ constraints, where each constraint corresponds to a subset $S$ of the elements. Intuitively, the constraint corresponding to subset $S$ has the following interpretation. Suppose that the elements in $S$ have been chosen to be included in the cover, contributing a total utility of $g(S)$. The assignment to the $x_{j}$ variables for the remaining elements designates which other elements should be added to $S$ to form a cover. The constraint says that the increases in utility that would be produced by adding each of these designated elements to $S$ individually, when added together, must be sufficient to achieve goal utility $Q$.

Wolsey proved that an assignment $x \in\{0,1\}^{n}$ to the variables in this IP is feasible if and only if $\left\{j \mid x_{j}=1\right\}$ is a cover for the associated SSC instance-that is, if and only if $g\left(\left\{j \mid x_{j}=1\right\}\right)=Q$. We call this Wolsey's property.

We present the dual of the LP relaxation of Wolsey's IP in Figure 2. This dual formulation was used by Fujito in his dual greedy algorithm for SSC and is the basis for ADG.

In Figure 3, we present a new LP, based on Wolsey's IP, which we call LP1. This LP generalizes Wolsey's IP for use in the binary SSSC problem. The LP incorporates $2^{n}$ different SSC problems, one for each possible combination of states for the $n$ items. The output of an algorithm for the SSSC problem is a decision tree (represented implicitly), which constructs a solution to each of the $2^{n}$ different SSC problems. LP1 does not enforce the restriction that the solutions must correspond to a tree, but a feasible solution to LP1 does obey a looser restriction, which we call the neighbor property.

Neighbor property: Let $T$ be a decision tree solving the SSSC problem. Given two assignments $a, a^{\prime} \in\{0,1\}^{n}$ differing only in bit $j$, either $T$ tests $j$ on both input $a$ and input $\alpha^{\prime}$, or on neither.

There are $n 2^{n-1}$ variables $x_{w}$ in LP1, one for each partial assignment $w$ such that $w_{j}=*$ for exactly one value of $j$. Let $W$ be the set of such assignments, so $W=\{w \in$ $\{0,1, *\}^{n} \mid w_{j}=*$ for exactly one value of $\left.j\right\}$. For $w \in W$, let $j(w)$ denote the $j \in N$ where 
$w_{j}=*$. Further, let $w^{(0)}$ and $w^{(1)}$ denote the extensions of $w$ obtained from $w$ by setting $w_{j(w)}$ to 0 and 1 , respectively. Finally, for $a \in\{0,1\}^{n}, j \in N$, let $a^{j}$ denote the partial assignment obtained from $a$ by setting $a_{j}$ to $*$. Note that for any fixed $a \in\{0,1\}^{n}$, the subset of constraints involving $a$, one for each $S \subseteq N$, is precisely the set of constraints of Wolsey's IP if we take the utility function to be $g_{a}$ such that $g_{a}(S)=g(S, a)$. Thus, a feasible solution to LP1 corresponds to the set of solutions for each of the $2^{n}$ individual SSC problems.

Lemma 3. The optimal value of LP1 lower bounds the expected cost of an optimal decision tree $T$ for the SSSC instance on $g, p$, and $c$.

Proof. Let $X$ be the assignment to the variables $x_{w}$ in the LP such that $x_{w}=1$ if $T$ tests $j$ on both assignments extending $w$ and $x_{w}=0$ otherwise. With respect to $X$, the expected cost of $T$ equals $\sum_{a \in\{0,1\}^{n}} \sum_{j} c_{j} x_{a^{j}} p(a)$. This equals the value of the objective function, because for $a, a^{\prime} \in\{0,1\}^{n}$ differing only in bit $j, p(a)+p\left(a^{\prime}\right)=p\left(a^{j}\right)$. Finally, as noted earlier, for any fixed $a \in\{0,1\}^{n}$, the subset of constraints involving $a$ is precisely the set of constraints of Wolsey's IP if we take the utility function to be $g_{a}$ such that $g_{a}(S)=g(S, a)$. Since $T$ produces a cover for every $a$, by Wolsey's property, the constraints of LP1 involving $a$ are satisfied. Thus, $X$ is a feasible solution to LP1, and the optimal value of the LP is at most the expected cost of the optimal tree.

We use our LP formulation to obtain a dual greedy algorithm-our ADG. We present the pseudocode for ADG in Algorithm 1. (In the line assigning a value to $j_{l}$, assume that if $E\left[g_{b}(x)\right]=0$, the expression evaluates to $\infty$.) Its main loop is analogous to the main loop in Fujito's dual greedy algorithm, except ADG uses expected increases in utility instead of known, deterministic increases in utility.

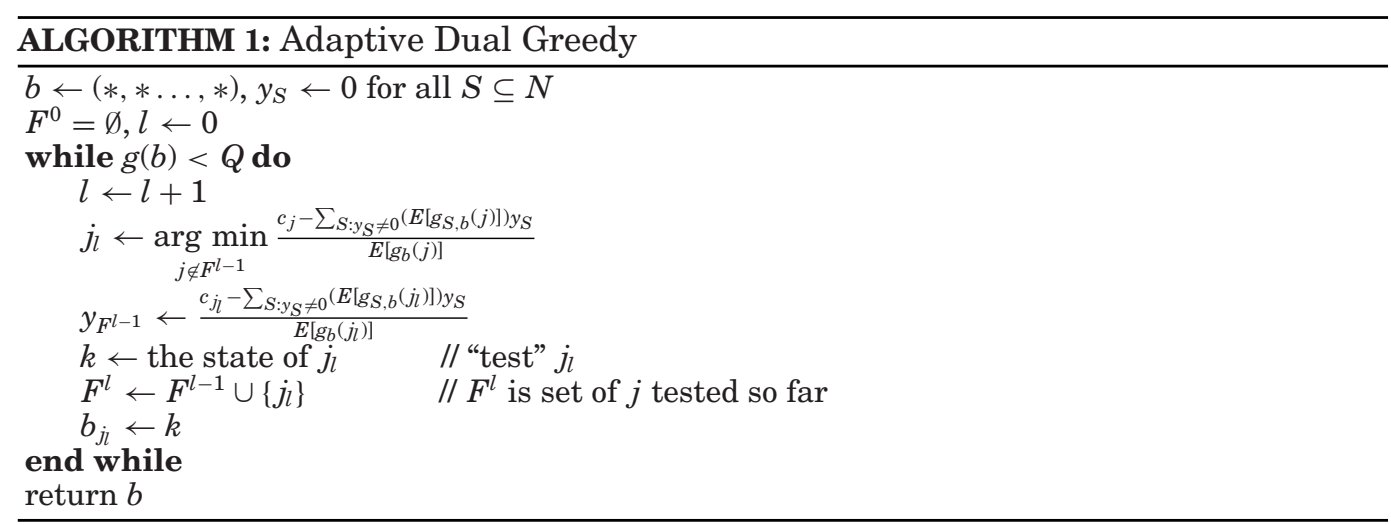

We now analyze ADG. To do this, we introduce a new LP that is closely related to the dual of LP1. To obtain this new LP, begin by taking each constraint in LP1 and multiplying both sides of the constraint by $p(a)$ (where $a, S$ is the pair corresponding to that constraint). Note that for all $w \in W$, variable $x_{w}$ appears only in the constraints for pairs $a, S$, where $a=w^{(0)}$ or $a=w^{(1)}$. Now take the dual of the modified LP and divide both sides of each constraint of the dual by $p(w)$ (where $w$ is the partial assignment corresponding to that constraint, and $j$ is such that $w_{j}=*$ ). Using the fact that $p\left(w^{(1)}\right) / p(w)=p_{j}$ and $p\left(w^{(0)}\right) / p(w)=1-p_{j}$, we get the new LP that we call $L P 2$, shown in Figure 4. (Note that although each in LP2 is shown as corresponding to a pair 


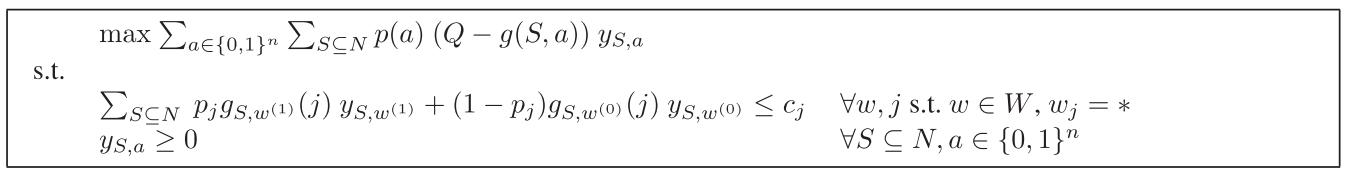

Fig. 4. LP2: the linear program for $\mathrm{ADG}$.

$w, j$, the condition that $w_{j}=*$ means that $j$ is uniquely determined by $w \in W$, and so each constraint actually corresponds uniquely to a $w \in W$.)

The variables in LP2 are $y_{S, a}$, where $S \subseteq N$ and $a \in\{0,1\}^{n}$.

Consider running ADG when $a \in\{0,1\}^{n}$ is the state vector that represents the initially unknown states of the items. Because $g(a)=Q, \mathrm{ADG}$ is guaranteed to terminate with an output $b$ such that $g(b)=Q$. Let $C(a)$ be the set of items that ADG tests and inserts into the cover it constructs for $a$. We will sometimes treat $C(a)$ as a sequence of items, ordered by their insertion order. ADG constructs an assignment to the variables $y_{S}$ (one for each $S \subseteq N$ ) when it is run on state vector $a$.

Consider the assignment $Y$ to the variables $y_{S, a}$ of LP2 such that each $y_{S, a}$ is given the value that ADG assigns to variable $y_{S}$ when it is run on state vector $a$. Let $Y_{S, a}$ denote the assignment that $Y$ makes to variable $y_{S, a}$.

We now show that $Y$ is a feasible solution to LP2 and that for each $a$, and each $j \in C(a), Y$ makes the constraint for $w=a^{j}$ tight. For $w \in W$, let $h_{w}^{\prime}(y)$ denote the function of the variables $y_{S, a}$ computed in the left-hand side of the constraint for $w$ in LP2.

Lemma 4. For every $a \in\{0,1\}^{n}, j \in N$,

(1) $h_{\alpha^{j}}^{\prime}(Y)=c_{j}$ if $j \in C(a)$ and

(2) $h_{a^{j}}^{\prime}(Y) \leq c_{j}$ if $j \notin C(a)$.

Proof. Assignment $Y$ assigns nonzero values only to variables $y_{S, a}$, where $S$ contains precisely the variables appearing in a prefix of sequence $C(a)$.

For $t \in N$, let $Y^{t}$ denote the assignment to the $y_{S, a}$ variables such that $y_{S, a}$ equals the value of variable $y_{S}$ at the end of iteration $t$ of the loop in ADG when it is run on state vector $a$. (If ADG terminates before iteration $t, y_{S, a}$ equals the final value of $y_{S}$.) Let $Y^{0}$ be the all 0 's assignment.

We first prove the following claim: for all $t \in N$ and $w \in W, h_{w}^{\prime}\left(Y^{t}\right)=$ $\sum_{S \subseteq N} E\left[g_{S, w}(j)\right] Y_{S, w^{(1)}}^{t}$.

Let $w \in W$. Consider running ADG on $w^{(0)}$ and $w^{(1)}$. Since it corresponds to a decision tree, the neighbor property holds. We consider two cases. For the first case, suppose that $j$ is never tested on $w^{(0)}$. Then it is never tested on $w^{(1)}$ and $Y_{S, w^{(0)}}^{t}=Y_{S, w^{(1)}}^{t}$ for all $S, t$. Thus, for all $t, h_{w}^{\prime}\left(Y^{t}\right)=\sum_{S \subseteq N}\left[p_{j} g_{S, w^{(1)}}(j)+\left(1-p_{j}\right) g_{S, w^{(0)}}(j)\right] Y_{S, w^{(1)}}^{t}=$ $\sum_{S \subseteq N} E\left[g_{S, w}(j)\right] Y_{S, w^{(1)}}^{t}$.

For the second case, suppose that $j$ is tested in iteration $\hat{t}$ on state vector $w^{(1)}$ and hence on state vector $w^{(0)}$. For $t \leq \hat{t}, Y_{S, w^{(1)}}^{t}=Y_{S, w^{(0)}}^{t}$ for all $S$. This is not true for $t>\hat{t}$. However, in iterations $t>\hat{t}, j$ is already part of the cover, so ADG assigns values only to variables $y_{S}$ where $j \in S$. For such $S, g_{S, w^{(1)}}(j)=0$. Thus, in this case also, $h_{w}^{\prime}\left(Y^{t}\right)=\sum_{S \subseteq N} E\left[g_{S, w}(j)\right] Y_{S, w^{(1)}}^{t}$ for all $t$. This completes the proof of the claim.

It is now easy to show by induction on $t$ that the two properties of the lemma hold for every $Y^{t}$ and hence for $Y$. They hold for $Y^{0}$. Assume that they hold for $Y^{t}$. Again consider assignments $w^{(1)}$ and $w^{(0)}$. If $j$ was tested on $w^{(1)}$ and $w^{(0)}$ in some iteration $\hat{t}<t+1$, then $h_{a^{j}}^{\prime}\left(Y^{t}\right)=h_{a^{j}}^{\prime}\left(Y^{t+1}\right)$ by the preceding arguments. If $j$ is tested in iteration 
$t+1$ on both state vectors, then the value assigned to $y_{F^{t}}$ by ADG on $w^{(1)}$ (and $w^{(0)}$ ) equals $\left(c_{j}-h_{w}^{\prime}\left(Y^{t}\right)\right) / E\left[g_{F^{l-1}, w}(j)\right]$, and thus $h_{w}^{\prime}\left(Y^{t+1}\right)=c_{j}$. If $j$ is not tested in iteration $t+1$ and was not tested earlier, the inductive assumption and the greedy choice criterion ensure that $h_{w}^{\prime}\left(Y^{t+1}\right) \leq c_{j}$.

The expected cost of the cover $C(a)$ produced by ADG on a random state vector $a$ is $\sum_{a \in\{0,1\}^{n}} \sum_{j \in C(a)} p(a) c_{j}$. The next lemma relates this value to an expression involving the $Y_{S, a}$.

Lemma 5. $\sum_{a \in\{0,1\}^{n}} \sum_{j \in C(a)} p(a) c_{j}=\sum_{a \in\{0,1\}^{n}} \sum_{S \subseteq N} \sum_{j: j \in C(a)} p(a) g_{S, a}(j) Y_{S, a}$

Proof. For $j \in N$, let $W^{j}=\left\{w \in W \mid w_{j}=*\right\}$. Then

$$
\begin{aligned}
& \sum_{a} \sum_{j: j \in C(a)} p(a) c_{j} \\
& =\sum_{j} \sum_{a: j \in C(a)} p(a) c_{j} \quad \text { switching the order of summation } \\
& =\sum_{j} \sum_{i \in\{0,1\}} \sum_{w \in W_{j}: j \in C\left(w^{(i)}\right)} p\left(w^{(i)}\right) c_{j} \quad \text { grouping assignments by the value of bit } j \\
& =\sum_{j} \sum_{i \in\{0,1\}} \sum_{w \in W_{j}: j \in C\left(w^{(0)}\right)}\left(p\left(w^{(i)}\right) c_{j}\right) \quad \text { by the neighbor property, } j \in C\left(w^{(1)}\right) \text { if and } \\
& =\sum_{j} \sum_{w \in W_{j}: j \in C\left(w^{(0)}\right)} p(w) c_{j} \quad \text { only if } j \in C\left(w^{(0)}\right) \\
& =\sum_{j} \sum_{w \in W_{j}: j \in C\left(w^{(0)}\right)} p(w) h_{w}^{\prime}(Y) \\
& =\sum_{j} \sum_{i \in\{0,1\}} \sum_{w \in W_{j}: j \in C\left(w^{(0)}\right)} \sum_{S} p\left(w^{(i)}\right) g_{S, w^{(i)}}(j) Y_{S, w^{(i)}} \\
& =\sum_{j} \sum_{i \in\{0,1\}} \sum_{w \in W_{j}: j \in C\left(w^{(i)}\right)} \sum_{S} p\left(w^{(i)}\right) g_{S, w^{(i)}}(j) Y_{S, w^{(i)}} \quad \text { because } j \in C\left(w^{(1)}\right) \text { if and only } \\
& =\sum_{j} \sum_{a: j \in C(a)} \sum_{S} p(a) g_{S, a}(j) Y_{S, a} \\
& =\sum_{a} \sum_{S} \sum_{j: j \in C(a)} p(a) g_{S, a}(j) Y_{S, a} \quad \text { if } j \in C\left(w^{(0)}\right)
\end{aligned}
$$$$
\text { only if } j \in C\left(w^{(0)}\right)
$$

switching the order of summation grouping assignments by the value of bit $j$

by Lemma 4 by the definition of $h_{w}^{\prime}$ because $j \in C\left(w^{(1)}\right)$ if and only if $j \in C\left(w^{(0)}\right)$

We can now prove the following theorem.

THEOREM 4. Given an instance of the binary SSSC problem with utility function $g$ and goal value $Q, A D G$ constructs a cover whose expected cost is no more than a factor of a larger than the expected cost of the cover produced by the optimal strategy, where $\alpha=\max \frac{\sum_{j \in C(a)} g_{S, a}(j)}{Q-g(S, a)}$, with the max taken over all pairs $S, a$, where $a \in\{0,1\}^{n}, S$ is a prefix of the cover $C(a)$ that $A D G$ constructs on state vector $a$, and $Q-g(S, a) \neq 0$.

Proof. By Lemma 5, the expected cost of the cover constructed by ADG is $\sum_{a} \sum_{S} \sum_{j: j \in C(a)} p(a) g_{S, a}(j) Y_{S, a}$. The value of the objective function of LP2 on $Y$ is $\sum_{a} \sum_{S} p(a)(Q-g(S, a)) Y_{S, a}$. For any $a, Y_{S, a}$ is nonzero if $S$ is a prefix of the cover that ADG constructs on state vector $a$. Comparing the coefficients of $Y_{S, a}$ in these two 
expressions implies that the expected cost of the cover is at most max $\frac{\sum_{j \in C(a)} g_{S, a}(j)}{Q-g(S, a)} \operatorname{times}$ the value of the objective function on $Y$. The theorem follows by Lemma 3 and weak duality.

The preceding analysis easily extends to the general SSSC problem, with a $k$-ary state space, yielding the same bound.

\section{ALGORITHMS FOR LINEAR THRESHOLD EVALUATION AND STOCHASTIC MIN-KNAPSACK}

Recall that an LTF with integer coefficients has the form $\sum_{i=1}^{n} a_{i} x_{i} \geq \theta$, where the $a_{i}$ and $\theta$ are integers. It represents the function $f:\{0,1\}^{n} \rightarrow\{0,1\}$ such that $f(x)=1$ if $\sum_{i=1}^{n} a_{i} x_{i} \geq \theta$ and $f(x)=0$ otherwise. We show how to use ADG to obtain an algorithm solving the SBFE problem for LTFs with integer coefficients.

Our results on ADG yield a 3-approximation for linear threshold evaluation.

THEOREM 5. There is a polynomial-time 3-approximation algorithm solving the SBFE problem for LTFs with integer coefficients.

Proof. Let $\sum_{i=1}^{n} a_{i} x_{i} \geq \theta$ be the LTF. Let $h(x)=\left(\sum_{i=1}^{n} a_{i} x_{i}\right)-\theta$. For $b \in\{0,1, *\}^{n}$, let $\min (b)$ be the minimum value of $h\left(b^{\prime}\right)$ for any extension $b^{\prime}$ of $b$. Similarly, let $\max (b)$ be the maximum value of $h\left(b^{\prime}\right)$ for any extension $b^{\prime}$ of $b$. Thus, $\min (b)=\left(\sum_{j: b_{j} \neq *} a_{j} b_{j}\right)+$ $\left(\sum_{i: a_{i}<0, b_{i}=*} a_{i}\right)-\theta, \max (b)=\left(\sum_{j: b_{j} \neq *} a_{j} b_{j}\right)+\left(\sum_{i: a_{i}>0, b_{i}=*} a_{i}\right)-\theta$, and each can be calculated in linear time. Let $R_{\min }=\min (*, \ldots, *)$ and $R_{\max }=\max (*, \ldots, *)$. If $R_{\min } \geq 0$ or $R_{\max }<0, f$ is constant and no testing is needed. Suppose that this is not the case.

Let $Q_{1}=-R_{\text {min }}$, and let submodular utility function $g_{1}$ be such that $g_{1}(b)=$ $\min \left\{-R_{\min }, \min (b)-R_{\min }\right\}$. Intuitively, if we consider the smallest and largest values of $h\left(b^{\prime}\right)$, for any $b^{\prime} \succeq b$ such that $f\left(b^{\prime}\right)=0$, then $Q_{1}-g_{1}(b)$ is the number of integers between these smallest and largest values, inclusive. Similarly, define $g_{0}(b)=\min \left\{R_{\max }+1, R_{\max }-\max (b)\right\}$ and $Q_{0}=R_{\max }+1$. Thus, $b$ is a 1-certificate of $f$ if and only if $g_{1}(b)=Q_{1}$ and a 0 -certificate if and only if $g_{0}(b)=Q_{0}$.

We apply the disjunctive construction of Lemma 1 to construct $g(b)=Q-\left(Q_{1}-\right.$ $\left.g_{1}(b)\right)\left(Q_{0}-g_{0}(b)\right)$, which is an assignment feasible utility function for $f$ with goal value $Q=Q_{1} Q_{0}$. We then run ADG using the utility functions and goal value defined earlier. By Theorem 4, the resulting algorithm is within a factor of $\alpha$ of optimal. We now show that $\alpha \leq 3$ in this case.

Fix $x$ and consider the run of ADG on $x$. Let $T$ be the number of loop iterations. Thus, $C(x)=j_{1}, \ldots, j_{T}$ is the sequence of tested items and $F^{t}=\left\{j_{1}, \ldots, j_{t}\right\}$. Assume first that $f(x)=1$. Let $F=F^{0}=\varnothing$, and consider the ratio $\frac{\sum_{j \in C(x)} g_{F, x}(j)}{Q-g(F, x)}$.

Assume without loss of generality that neither $g_{0}$ nor $g_{1}$ is identically 0 .

Let $A=-R_{\text {min }}$, and let $B=R_{\max }+1$. Thus, $Q-g(\emptyset, x)=A B$. Let $C_{1}$ be the set of items $j_{l}$ in $C(x)$ such that either $x_{j_{l}}=1$ and $a_{j_{l}} \geq 0$ or $x_{j_{l}}=0$ and $a_{j_{l}}<0$. Similarly, let $C_{0}$ be the set of items $j_{l}$ in $C(x)$ such that either $x_{j_{l}}=0$ and $a_{j_{l}} \geq 0$ or $x_{j_{l}}=1$ and $a_{j_{l}}<0$.

Testing stops as soon as the goal utility is reached. Since $f(x)=1$, this means that testing on $x$ stops when $b$ satisfies $g_{1}(b)=Q_{1}$, or equivalently, $b$ is a 1 -certificate of $f$. Thus, the last tested item, $j_{T}$, is in $C_{1}$. Further, the sum of the $a_{j l} x_{j l}$ over all $j_{l} \in C_{1}(x)$, excluding $j_{T}$, is less than $-R_{\text {min }}$, whereas the sum including $j_{T}$ is greater than or equal to $-R_{\text {min }}$. By the definition of utility function $g, \sum_{j_{l}: j_{l} \in C_{1}: j_{l} \neq j_{T}} g_{\emptyset, x}\left(j_{l}\right)<A B$. The maximum possible value of $g_{\emptyset, x}\left(j_{T}\right)$ is $A B$. Therefore, $\sum_{j_{l}: j_{l} \in C_{1}} g_{\emptyset, x}\left(j_{l}\right)<2 A B$. 
Since $x$ does not contain both a 0 -certificate and a 1-certificate of $f$, the sum of the $a_{j_{l}} x_{j_{l}}$ over all $j_{l}: j_{l} \in C_{0}(x)$ is strictly less than $R_{\text {max }}$. Thus, by the definition of $g$, $\sum_{j_{l}: j_{l} \in C_{0}} g_{\emptyset, x}\left(j_{l}\right)<A B$. Summing over all $j_{l}: j_{l} \in C(x)$, we get that $\sum_{j_{l}: j_{l} \in C(x)} g_{\emptyset, x}\left(j_{l}\right)<$ $3 A B$. Therefore, for $F=\emptyset, \frac{\sum_{j \in C(x)} g_{F, x}(j)}{Q-g(F, x)}<3$ because $Q=A B$ and $g(\emptyset, x)=0$. A symmetric argument holds when $f(x)=0$.

It remains to show that the same bound holds when $F \neq \emptyset$. We reduce this to the case $F=\emptyset$. Once we have tested the variables in $F^{t}$, we have an induced linear threshold evaluation problem on the remaining variables (replacing the tested variables by their values). Let $g^{\prime}$ and $Q^{\prime}$ be the utility function and goal value for the induced problem. The ratio $\frac{\sum_{j: j \in C(x)} g_{F, x}(j)}{Q-g(F, x)}$ is equal to $\frac{\sum_{j: j \in C(x)-F} g_{\square, x^{\prime}}^{\prime}(j)}{Q-g^{\prime}\left(\emptyset, x^{\prime}\right)}$, where $x^{\prime}$ is $x$ restricted to the elements not in $F$. By the preceding argument, this ratio is bounded by 3 .

We can use essentially the same approach to obtain results for stochastic minknapsack.

THEOREM 6. There is a polynomial-time 3-approximation algorithm solving the stochastic min-knapsack problem. For the variant of stochastic min-knapsack where threshold $\theta$ can always be reached or exceeded, there is a polynomial-time 2approximation.

Proof. Given a stochastic min-knapsack instance, we can associate with it the LTF $x_{1}+x_{2}+\cdots+x_{n} \geq \theta$, over items $\{1, \ldots, n\}$, where each item $i$ has cost $c_{i}$ and $x_{i}$ is $s_{i}$, the size of item $i$. Without loss of generality, we can assume that the size $s_{i}$ of every item is at most $\theta$.

Placing items in the knapsack until the sum of the sizes of the chosen items is at least $\theta$ is like testing the values of the variables in this LTF until the formula evaluates to $\theta$. We would therefore like to apply the proof of the 3-approximation bound for LTFs to stochastic min-knapsack. However, we need to modify the proof slightly to account for differences between the threshold evaluation problem and stochastic min-knapsack.

The main difference is how the two problems deal with the situation where the threshold value cannot be attained. In linear threshold evaluation, we can stop testing as soon as we have a 0-certificate, but in min-knapsack, we must pay to put all of the items in the knapsack if the threshold is not reached. To account for this difference, we modify the utility function. Let $g_{1}(b)=\min \left(\sum_{i: b_{i} \neq *} b_{i}, \theta\right)$ with goal $Q_{1}=\theta$, and $g_{0}(b)=$ $\left|\left\{i: b_{i} \neq *\right\}\right|$ with goal $Q_{0}=n$. Combining these with the disjunctive construction, we get utility function $g$ with goal value $Q=n \theta$. We can solve the stochastic min-knapsack problem by solving the SSSC problem for $g$ and $Q$.

Another difference is that for linear threshold evaluation, we considered only Bernoulli distributions on the variables $x_{i}$. Since in stochastic min-knapsack, we assumed that each $s_{i}$ has a finite number of possible values, we just have a $k$-ary SSSC problem for some $k$ rather than a binary one. Thus, the $\alpha$ bound on ADG still holds.

We show that $\alpha \leq 3$ when ADG is run on $g$. As in the proof for linear threshold, it suffices to show that $\sum_{j=1}^{k} g_{\emptyset, s}(j) /(Q-g(s, \emptyset)) \leq 3$ for all possible size vectors $s$.

We first consider the case where the threshold is achieved by $s$ - that is, $\sum_{j=1}^{n} s_{j} \geq \theta$. Suppose that we run ADG with size vector $s$, and without loss of generality we test items $1, \ldots, k$, in that order. For $j \in\{1, \ldots, k\}$, the contribution to $g_{0}$ for the $j$ th item (if $j$ is added by itself to $\emptyset$ ) is 1 , and the contribution to $g_{1}$ is $s_{j}$. Thus, for each $j \in\{1, \ldots k\}$, $g_{\emptyset, s}(j)=\theta+s_{j}(n-1)$. Further, $\sum_{j=1}^{k-1} s_{j}<\theta$, and since each $s_{i} \leq \theta$ by assumption, $\sum_{j=1}^{k} g_{\emptyset, s}(j) \leq \theta(k+2(n-1))<3 n \theta$, and therefore $\sum_{j=1}^{k} g_{\emptyset, s}(j) /(Q-g(s, \emptyset)) \leq 3$. 
We now consider the case where the threshold is not achieved on $s$. In this case, $\sum_{j=1}^{k} s_{j}<\theta$, and $\sum_{j=1}^{k} g_{\emptyset, s}(j) \leq(k+(n-1)) \theta<2 n \theta$. Therefore, $\sum_{j=1}^{k} g_{\emptyset, s}(j) /(Q-$ $g(s, \emptyset)) \leq 2$. This inequality also holds if we replace $\emptyset$ by any prefix of $\{1, \ldots, k\}$. Thus, $\alpha<3$, and we have a 3-approximation algorithm.

In the variant of stochastic min-knapsack where the distributions on the sizes are such that we are guaranteed to to reach the threshold of $\theta$, we can take utility function $g$ to be just the $g_{1}$ defined earlier. It is easy to show that $\alpha \leq 2$ for this $g$, and we have a 2-approximation algorithm.

\section{A NEW BOUND FOR THE ADAPTIVE GREEDY ALGORITHM}

We give a new analysis of the adaptive greedy algorithm of Golovin and Krause, whose pseudocode we present in Algorithm 2. We note that the analysis provided by Golovin and Krause applied to a more general setting, in which the utility function and the distribution on states jointly satisfy a property called adaptive submodularity. Our analysis does not apply to this more general setting.

Some of the variables used in the pseudocode are not necessary for the running of the algorithm but are useful in its analysis. (In the line assigning a value to $j$, assume that if $E\left[g_{b}(j)\right]=0$, the expression evaluates to $\infty$.)

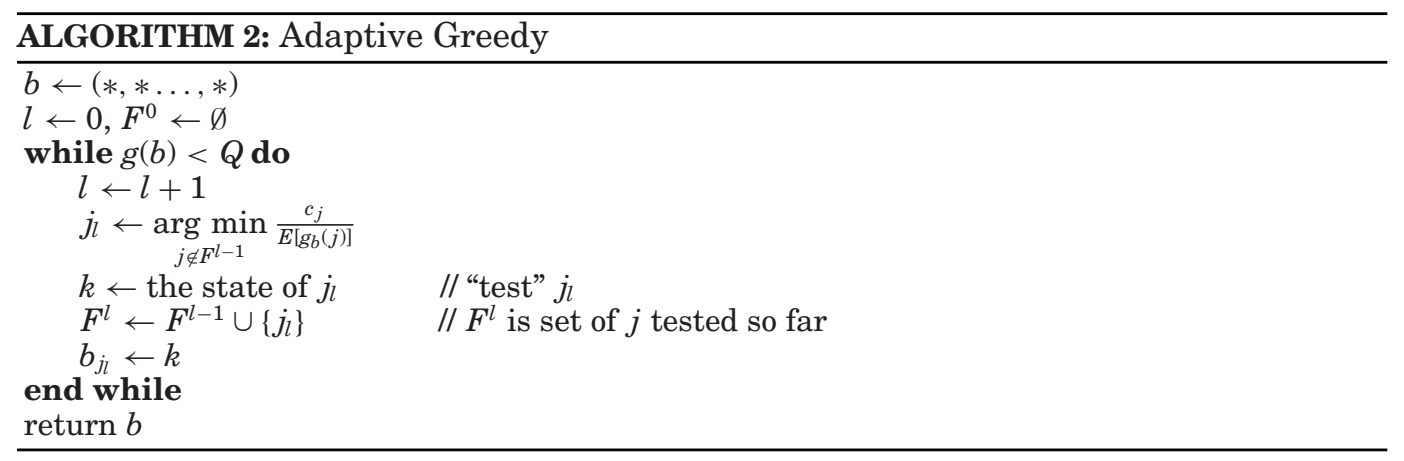

Throughout this section, we let $g(j)=\max _{l \in \mathcal{O}} g_{r}(j, l)$, where $r=(*, \ldots, *)$ and $\mathcal{O}$ is the state space. Thus, $g(j)$ is the maximum increase in utility that can be obtained as a result of testing $j$ (since $g$ is submodular).

Unless otherwise noted, assume that $\mathcal{O}=\{0,1\}$.

The adaptive greedy algorithm is a natural extension of Wolsey's greedy algorithm for (deterministic) SSC. We will extend Wolsey's analysis [Wolsey 1982] as it was presented by Fujito [2000]. In our analysis, we will refer to LP2, defined in Section 6, along with the associated notation for the constraints $h_{a^{j}}^{\prime}(y) \leq c_{j}$.

For $x \in\{0,1\}^{n}$, let $T^{x}$ be the number of iterations of the while loop in the adaptive greedy algorithm on state vector $x$. Let $b_{x}^{t}$ denote the value of $b$ at the end of iteration $t$ of the while loop on state vector $x$, and let $F_{x}^{t}$ denote the value of $F^{t}$, where $F^{t}$ is the set of $j$ s tested by the end of the $t+1$ st iteration. (The $x$ in the notation may be dropped when it is understood implicitly.) Set $\theta_{x}^{t}=\min _{j \notin F^{t-1}} \frac{c_{j}}{E\left[g_{b}^{t-1}(j)\right]}$.

To analyze the adaptive greedy algorithm, we define $Y$ to be the assignment to the LP2 variables $y_{S, x}$ setting $y_{F^{0}, x}=\theta_{x}^{1}, y_{F^{t}, x}=\left(\theta_{x}^{t+1}-\theta_{x}^{t}\right)$ for $t \in\left\{1 \ldots T^{x}-1\right\}$ and $y_{S, x}=0$ for all other $S$. We define $Y^{x}$ to be the restriction of that assignment to the variables $y_{S, x}$ for that $x$. Let $q^{x}(y)=\sum_{S \subset N}(Q-g(S, x)) y_{S, x}$.

LEMMA 6. The expected cost of the cover constructed by the adaptive greedy algorithm is at most $E\left[q^{x}\left(Y^{x}\right)\right]$, where the expectation is with respect to $x \sim D_{p}$. 
Proof. By the definition of $Y^{x}$, the proof follows directly from the analysis of (nonadaptive) greedy in Theorem 1 of Fujito [2000] by linearity of expectation.

We need to bound the value of $h_{w}^{\prime}(Y)$ for each $w \in W$, where $h_{w}^{\prime}(Y)$ again refers to the function of the variables $y_{S, a}$ computed in the left-hand side of the constraint for $w$ in LP2.

We will use the following lemma from Wolsey's analysis.

Lemma 7. Wolsey [1982] Given two sequences $\left(\alpha^{(t)}\right)_{t=1}^{T}$ and $\left(\beta^{(t)}\right)_{t=0}^{T-1}$ such that both are nonnegative, the former is monotonically nondecreasing and the latter is monotonically nonincreasing, and $\beta^{(t)}$ is a nonnegative integer for any value of $t$, then

$$
\begin{gathered}
\alpha^{(1)} \beta^{(0)}+\left(\alpha^{(2)}-\alpha^{(1)}\right) \beta^{(1)}+\cdots+\left(\alpha^{(T)}-\alpha^{(T-1)}\right) \beta^{(T-1)} \\
\leq\left(\max _{1 \leq t \leq T} \alpha^{(t)} \beta^{(t-1)} H\left(\beta^{(0)}\right)\right),
\end{gathered}
$$

where $H(n)$ denotes the nth harmonic number, which is at most $(\ln n+1)$.

Let $r$ denote the assignment $(*, \ldots, *)$, and let $H_{j}^{1}=H\left(g_{r}(j, 1)\right)$ and $H_{j}^{0}=H\left(g_{r}(j, 0)\right)$.

Lemma 8. For every $x \in\{0,1\}^{n}$ and $j \in\{1, \ldots, N\}, h_{x^{j}}^{\prime}(Y) \leq c_{j} 2 H\left(\max _{i \in N} g(i)\right)$.

Proof. We fix $x \in\{0,1\}^{n}$ and $j \in N$. Let $x^{\prime}$ be the assignment obtained from $x$ by complementing $x_{j}$. Without loss of generality, assume that $x_{j}=1$ (and $x_{j}^{\prime}=0$ ).

Let $k$ be the value of $t$ that maximizes $\left(\theta_{x}^{t}\right)\left(g_{b_{x}^{t-1}}(j, 1)\right)$. Similarly, let $l$ be the value of $t$ that maximizes $\left(\theta_{x^{\prime}}^{t}\right)\left(g_{b_{x^{\prime}}^{t-1}}(j, 0)\right)$. Let $q_{j}=1-p_{j}$.

By the submodularity of $g$, and the greedy choice criterion used by the adaptive greedy algorithm, $\theta_{x}^{1} \leq \theta_{x}^{2} \ldots \leq \theta_{x}^{T^{x}}$. By the submodularity of $g, g_{b_{x}^{0}}(j, 0) \geq g_{b_{x}^{1}}(j, 0) \ldots \geq$ $g_{b_{x}^{T x-1}}(j, 0)$. Thus, Lemma 7 applies to the nondecreasing sequence $\theta_{x}^{1}, \theta_{x}^{2}, \ldots \theta_{x}^{T^{x}}$ and the nonincreasing sequence $g_{b_{x}}^{0}(j, 0), g_{b_{x}}^{1}(j, 0), \ldots, g_{b_{x}}^{T^{x}}-1(j, 0)$. This also holds if we substitute $(j, 1)$ for $(j, 0)$ in the second sequence.

Then,

$$
\begin{aligned}
& h_{x^{j}}^{\prime}(Y) \\
& =\sum_{S \subseteq N}\left(p_{j} g_{S, x}(j)+\left(1-p_{j}\right) g_{S, x^{\prime}}(j)\right) Y_{S, x} \\
& =\begin{array}{r}
p_{j}\left[\theta_{x}^{1} g_{b_{x}^{0}}(j, 1)+\Sigma_{i=2}^{T^{x}}\left(\theta_{x}^{i}-\theta_{x}^{i-1}\right) g_{b_{x}^{i-1}}(j, 1)\right]+ \\
{\left[\theta_{x^{\prime}}^{1} g_{b_{x^{\prime}}^{0}}(j, 0)+\Sigma_{i=2}^{T^{x^{\prime}}}\left(\theta_{x^{\prime}}^{i}-\theta_{x^{\prime}}^{i-1}\right) g_{b_{x^{\prime}}^{i-1}}(j, 0) q_{j}\right]}
\end{array} \\
& \leq p_{j}\left[\theta_{x}^{k} g_{b_{x}^{k-1}}(j, 1) H_{j}^{1}\right]+q_{j}\left[\theta_{x^{\prime}}^{l} g_{b_{x^{\prime}}^{l-1}}(j, 0) H_{j}^{0}\right] \\
& \leq \begin{array}{r}
p_{j}\left[\theta_{x}^{k} g_{b_{x}^{k-1}}(j, 1) H_{j}^{1}\right]+q_{j}\left[\theta_{x}^{k} g_{b_{x}^{k-1}}(j, 0) H_{j}^{1}\right]+ \\
p_{j}\left[\theta_{x^{\prime}}^{l} g_{b^{l-1}}(j, 1) H_{j}^{0}\right]+q_{j}\left[\theta_{x^{\prime}}^{l} g_{b_{x^{\prime}}^{l-1}}(j, 0) H_{j}^{0}\right]
\end{array} \\
& \text { since this just adds extra nonnegative terms } \\
& =\begin{array}{r}
\theta_{x}^{k} H_{j}^{1}\left[p_{j} g_{b_{x}^{k-1}}(j, 1)+q_{j} g_{b_{x}^{k-1}}(j, 0)\right]+ \\
\theta_{x^{\prime}}^{l} H_{j}^{0}\left[q_{j} g_{b_{x^{\prime}}^{l-1}}(j, 0)+p_{j} g_{b_{x^{\prime}}^{l-1}}(j, 1)\right]
\end{array} \\
& \leq c_{j} H_{j}^{1}+c_{j} H_{j}^{0} \\
& \leq c_{j} 2 H(g(j)) \\
& \leq c_{j} 2 H\left(\max _{i} g(i)\right) \text {. }
\end{aligned}
$$


THEOREM 7. Given an instance of the binary SSSC problem with utility function $g$, the adaptive greedy algorithm constructs a decision tree whose expected cost is no more than a factor of $2\left(\max _{i \in N}(\ln g(i))+1\right)$ larger than the expected cost of the cover produced by the optimal strategy.

PROOF. Let OPTDT be the expected cost of the cover produced by the optimal strategy (decision tree). Let AGCOST be the expected cost of the cover produced by the adaptive greedy algorithm, and let $q(y)$ denote the objective function of LP2. By Lemma 3, the optimal value of LP1 is a lower bound on OPTDT. Let $Z=Y /\left(2 H\left(\max _{i} g(i)\right)\right)$. By Lemma $8, Z$ is a feasible solution to LP2. Thus, by weak duality, $q(Z) \leq$ OPTDT. By Lemma 6 , AGCOST $\leq E\left[q^{x}\left(Y^{x}\right)\right]$, and it is easy to see that $E\left[q^{x}\left(Y^{x}\right)\right]=q(Y)$. Since $q(Y)=q(Z)\left(2 H\left(\max _{i} g(i)\right)\right)$, AGCOST $\leq 2 H\left(\max _{i} g(i)\right)$ OPTDT.

The preceding analysis can be generalized to the $k$-ary SSSC problem, but the bound becomes $k\left(\max _{i \in N}(\ln g(i))+1\right)$. The $k$ in this bound results from the generalization of Lemma 8.

Note that $\max _{j} g(j)$ is clearly upper bounded by $Q$ and in some instances may be much less than $Q$. However, because of the factor of $k$ at the front of our bound, we cannot say that it is strictly better than the $(\ln Q+1)$ bound of Golovin and Krause. We do not know whether this dependence on $k$ is necessary.

\section{APPROXIMATION BOUNDS IN TERMS OF THE EXPECTED COST OF COVER}

The approximation bounds that we have shown for the adaptive greedy algorithm and ADG compare the expected cost of the constructed cover to the expected cost of the cover produced by the optimal decision tree. As before, call the latter quantity OPTDT. We are also interested in comparing the adaptive greedy algorithm and ADG to another quantity, which we call OPTCOVER. The quantity OPTCOVER is the expected cost, over all $x$, of the cost of the minimum-cost cover for $x$. Clearly, OPTCOVER $\leq$ OPTDT.

By Lemma 3, the optimal value of LP1 for a given SSSC instance lower bounds the value of OPTDT for that instance. Let OPTLP denote the optimal value of LP1.

THEOREM 8. For any instance of the binary SSSC problem over the uniform distribution on $\{0,1\}^{n}, O P T L P \leq 2(O P T C O V E R)$.

Proof. Associate with each assignment $a \in\{0,1\}^{n}$ a minimum-cost cover $C$ for $a$. Call this cover $C(a)$. Then OPTCOVER is the expected cost of $C(a)$ when $a$ is a uniformly random assignment.

We say that these covers obey the neighbor property if for any two assignments $a$ and $a^{\prime}$ differing in a single bit $j$, either $j$ is in both $C(a)$ and $C\left(a^{\prime}\right)$ or $j$ is in neither.

If the covers do not obey the neighbor property, modify the covers using the following procedure to cause the property to hold: for each of the $n 2^{n}$ ordered pairs of assignments $\left(a, a^{\prime}\right)$ differing in a single bit $j$, if $j$ is in $C(a)$ but not in $C\left(a^{\prime}\right)$, add $j$ to $C\left(a^{\prime}\right)$.

We now show that the preceding modification increases the expected cover cost by at most a factor of 2 using a simple amortization argument. For each pair of assignments $a$ and $a^{\prime}$ that differed only in a single bit $j$ appearing in $C(a)$ but not in $C\left(a^{\prime}\right)$, let the cost of adding $j$ to $C\left(a^{\prime}\right)$ be "charged" to the cost of including $j$ in $C(a)$. Under the uniform distribution, this at most doubles the cost of $C(a)$. Thus, the expected cover cost, after the preceding modification, is at most 2(OPTCOVER).

Now construct an assignment $X$ to the variables $x_{w}$ in LP1 as follows, using the modified covers. If $j$ is the bit on which $w_{j}=*$, and $a$ and $a^{\prime}$ are the two assignments extending $w$, set $x_{w}=1$ if $j$ is in both $C(a)$ and $C\left(a^{\prime}\right)$, and set $x_{w}=0$ otherwise. Because the variables $x_{w}$ set to 1 by $X$ correspond to covers for all $a \in\{0,1\}^{n}$, the constraints of LP1 are satisfied and $X$ is a feasible solution to LP1. The value of the objective 
function for LP1 on $X$ is the expected cover cost, which is at most 2(OPTCOVER). Thus, OPTLP $\leq 2$ (OPTCOVER).

We can generalize the preceding theorem to apply to product distributions. Define $\rho$ to denote, over all $i$, the minimum $p_{i}$ or the minimum $1-p_{i}$, whichever is smaller. In the proof, we can change the amortization argument to charge $\frac{1}{p_{j}}$ or $\frac{1}{1-p_{j}}$ (depending on the value of $a_{j}$ ) for the items $j$ that are in $C(a)$ and added to $C\left(a^{\prime}\right)$. This yields the following corollary.

Corollary 1. For any instance of the binary SSSC problem, OPTLP $\leq \frac{1}{\rho}$ (OPTCOVER).

The preceding can be easily generalized to the $k$-ary SSSC problem. For $l \in \mathcal{O}$ and $i \in\{1, \ldots, n\}$, let $p_{i, l}=\operatorname{Prob}\left[x_{i}=l\right]$, and let $\rho$ be the minimum value of the $p_{i, l}$. Extending the preceding argument again yields the bound OPTLP $\leq \frac{1}{\rho}$ (OPTCOVER).

In the previous section, we proved a new bound for the adaptive greedy algorithm that achieves a solution for the binary SSSC problem that is within a factor of $2(\ln P+1)$ of OPTLP, where $P$ is the maximum utility that can be contributed by a single item. Since $P \leq Q$, by Lemma 8 , the adaptive greedy algorithm produces a solution that is within a factor of $4(\ln Q+1)$ of OPTCOVER under the uniform distribution. Similarly, ADG produces a solution that is within a factor of $2 \alpha$ of OPTCOVER under the uniform distribution.

We now revisit the $Q$-value approach to the SBFE problem presented in Section 5.1. We stated there that creating an assignment-feasible utility function $g$ for the SBFE problem on function $f$, and running the adaptive greedy algorithm using $g$, achieves an approximation factor of $\ln Q+1$ with respect to the optimal decision tree for $f$. Let OPTCERT denote the expected cost of the optimal certificate for an SBFE problem on a given function $f$. Since OPTCOVER for $g$ is equal to OPTCERT for $f$ here, it follows that under the uniform distribution, the $Q$-value approach for the SBFE problem yields a solution that is within a factor of $4(\ln Q+1)$ of OPTCERT.

Previously, we used the $Q$-value approach on the CDNF evaluation problem to achieve an approximation factor of $(\ln k d+1)$ with respect to the optimal decision tree, where $k$ is the number of terms and $d$ is the number of clauses. It follows from the preceding that under the uniform distribution, the $Q$-value approach achieves an approximation factor of $4(\ln k d+1)$ with respect to OPTCERT. This bound is within a factor of 2 of the bound previously achieved by Kaplan et al. [2005], also with respect to OPTCERT, for the special case of monotone CDNF with unit costs. The $O(\log k d)$ approximation factor is, in fact, nearly optimal for CDNF evaluation with respect to OPTCERT [Allen et al. 2013].

\section{SIMULTANEOUS EVALUATION AND RANKING}

Let $f_{1}, \ldots, f_{m}$ be (representations of) Boolean functions from a class $C$ such that each $f_{k}:\{0,1\}^{n} \rightarrow\{0,1\}$. We consider the generalization of the SBFE problem where instead of determining the value of a single function $f$ on an input $x$, we need to determine the value of all $m$ functions $f_{k}$ on the same input $x$.

The $Q$-value approach can be easily extended to this problem by constructing utility functions for each of the $f_{i}$ and combining them using the conjunctive construction in Lemma 1. The algorithm of Golovin and Krause [2011] for simultaneous evaluation of OR formulas follows this approach (Liu et al. [2008] presented a similar algorithm earlier using a different analysis.) We can also modify the approach by calculating a bound based on the $P$-value or using ADG instead of the adaptive greedy algorithm. We thus obtain the following theorem, where $\sum_{i=1}^{n} a_{k_{i}} x_{i} \leq \theta_{k}$ is the $k$ th threshold formula. 
THEOREM 9. There is a polynomial-time algorithm for solving the problem of simultaneous evaluation of LTFs that produces a solution that is within a factor of $O\left(\log m D_{\text {avg }}\right)$ of optimal, where $D_{\text {avg }}$ is the average, over $k \in\{1, \ldots, m\}$, of $\sum_{i=1}^{n}\left|a_{k_{i}}\right|$. In the special case of $O R$ formulas, where each variable appears in at most $r$ of them, the algorithm achieves an approximation factor of $2\left(\ln \left(\beta_{\max } r\right)+1\right)$, where $\beta_{\text {max }}$ is the maximum number of variables in any of the OR formulas.

There is also a polynomial-time algorithm for solving the problem of simultaneous evaluation of LTFs that produces a solution that is within a factor of $D_{\max }$ of optimal, where $D_{\text {max }}=\max _{k \in\{1, \ldots, m\}} \sum_{i=1}^{n}\left|a_{k_{i}}\right|$.

PRoOF. We use the utility function construction for LTFs from the beginning of the proof of Theorem 1. Let $g^{(1)}, \ldots, g^{(m)}$ be the $m$ utility functions that result from applying this construction to the $m$ LTFs that need to be evaluated. Let $Q^{(1)}, \ldots, Q^{(m)}$ be the associated goal values.

Using the conjunctive construction from Lemma 1, we construct utility function $g$ such that $g(b)=\sum_{k=1}^{m} g^{(k)}(b)$, and $Q=\sum_{k=1}^{m} Q^{(k)}$.

To obtain the first algorithm, we evaluate all threshold formulas by running the adaptive greedy algorithm with $g$, goal value $Q$, and the given $p$ and $c$ until it outputs a cover $b$. Given cover $b$, it is easy to determine the value of $f_{k}(x)$ for each $f_{k}$.

For each $f_{k}$, the associated $Q^{(k)}$ is at most $D_{k}^{2}$, where $D_{k}=\sum_{i=1}^{n}\left|a_{k_{i}}\right|$. Because $Q=$ $\sum_{k} Q^{(k)}$ and $\sum_{k} D_{k}^{2} \leq\left(\sum_{k} D_{k}\right)^{2}=\left(m D_{\text {avg }}\right)^{2}$, the $O\left(\log \left(m D_{\text {avg }}\right)\right)$ bound follows from the $(\ln Q+1)$ bound for the adaptive greedy algorithm.

Suppose that each threshold formula is an OR formula. For $b \in\{0,1, *\}^{n}$, $\max _{l \in\{0,1\}} g_{b}^{(k)}(j, l)=0$ if $x_{j}$ does not appear in the $k$ th OR formula; otherwise, it is equal to the number of variables in that formula. The $2\left(\ln \left(\beta_{\max } r\right)+1\right)$-approximation factor then follows by our bound on the adaptive greedy algorithm in Theorem 7 .

For the second algorithm, we just use ADG instead of the adaptive greedy algorithm with the same utility function $g$. The approximation factor for ADG, given in Theorem 4, is upper bounded by $\max _{S} \frac{\sum_{j} g_{S, x}(j)}{Q-g(S, x)}$, where $S$ is any subset of $\{1, \ldots, n\}$.

We first bound this ratio for $S=\emptyset$. We have $\frac{\sum_{j} g_{\emptyset, x}(j)}{Q-g(\emptyset, x)}=\frac{\sum_{k} \sum_{j} g_{\emptyset, x}^{(k)}(j)}{\sum_{k} Q^{(k)}}$. This quantity is bounded above by the maximum, over $k$, of the quantity $\frac{\sum_{j} g_{(x, x}^{(k)}(j)}{Q^{(k)}}$.

By the construction of the utility functions, $\frac{g_{0, x}^{(k)}(j)}{Q^{(k)}} \leq\left|a_{k_{j}}\right|$. It follows that $\frac{\sum_{j} g_{Q_{x}}^{(k)}(j)}{Q^{(k)}} \leq$ $\sum_{j}\left|a_{k_{j}}\right|$, and therefore $\frac{\sum_{j} g_{\emptyset, x}(j)}{Q-g(\emptyset, x)} \leq D_{\max }$.

Suppose that $S \neq \emptyset$. For each $f_{k}$, fix the variables $j \in S$ according to their values in $x$. This yields a new LTF $f_{k}^{\prime}$ with associated utility function $g_{k}^{\prime}$ and goal value $Q_{k}^{\prime}$. The terms of $f_{k}^{\prime}$ are a subset of the terms of $f_{k}$ (the threshold may be different). Letting $x^{\prime}$ be the restriction of $x$ to the variables not in $S$, we have that $g_{S, x}(j)=g_{\emptyset, x^{\prime}}^{\prime}(j)$ for $j \notin S$. It therefore follows from the preceding that $\frac{\sum_{j} g_{S, x}(j)}{Q-g(S, x)} \leq D_{\max }^{\prime}$, where $D_{\max }^{\prime}$ is the value analogous to $D_{\max }$ for the $f_{k}^{\prime}$. Since the terms of $f_{k}^{\prime}$ are a subset of the terms of $f_{k}$, $D_{\max }^{\prime} \leq D_{\max }$.

For the special case of simultaneous evaluation of OR formulas, the theorem implies a $\beta$-approximation algorithm, where $\beta$ is the length of the largest $\mathrm{OR}$ formula. This improves the $2 \beta$-approximation achieved by the randomized algorithm of Liu et al. [2008].

We use a similar approach to solve the linear function ranking problem. In this problem, you are given a system of linear functions $f_{1}, \ldots, f_{m}$, where for $j \in\{1, \ldots, m\}$, $f_{j}$ is $a_{j_{1}} x_{1}+a_{j_{2}} x_{2}+\cdots a_{j_{n}} x_{n}$ and the coefficients $a_{j_{i}}$ are integers. You would like to 
determine the sorted order of the values $f_{1}(x), \ldots, f_{m}(x)$ for an initially unknown $x \in$ $\{0,1\}^{n}$. (Note that the values of the $f_{j}(x)$ are not Boolean.) We consider the problem of finding an optimal testing strategy for this problem, where as usual, $x \sim D_{p}$ for some probability vector $p$, and there is a cost vector $c$ specifying the cost of testing each variable $x_{i}$.

Note that there may be more than one correct output for this problem if there are ties. Thus, strictly speaking, this is not a function evaluation problem. Nevertheless, we can still exploit our previous techniques. For each system of linear equations $f_{1}, \ldots, f_{m}$ over $x_{1}, \ldots, x_{n}$, and each $x \in\{0,1\}^{n}$, let $f(x)$ denote the set of permutations $\left\{f_{j_{1}}, f_{j_{2}}, \ldots, f_{j_{m}}\right\}$ of $f_{1}, \ldots, f_{m}$ such that $f_{j_{1}}(x) \leq f_{j_{2}}(x) \leq \cdots \leq f_{j_{m}}(x)$. The goal of sorting the $f_{j}$ is to output some permutation that we know definitively to be in $f(x)$. Note that in particular, if for example $f_{i}(x)<f_{j}(x)$, it may be enough for us to determine that $f_{i}(x) \leq f_{j}(x)$.

THEOREM 10. There is an algorithm that solves the linear function ranking problem that runs in time polynomial in $m, n$, and $D_{\max }$ and achieves an approximation factor that is within $O\left(\log \left(m D_{\max }\right)\right)$ of optimal, where $D_{\max }$ is the maximum value of $\sum_{i=1}^{n}\left|a_{j_{i}}\right|$ over all the functions $f_{j}$.

Proof. For each pair of linear equations $f_{i}$ and $f_{j}$ in the system, where $i<j$, let $f_{i j}$ denote the linear function $f_{i}-f_{j}$. We construct a utility function $g^{(i j)}$ with goal value $Q^{(i j)}$. Intuitively, the goal value of $g^{(i j)}$ is reached when there is enough information to determine that $f_{i j}(x) \geq 0$, or when there is enough information to determine that $f_{i j}(x) \leq 0$.

For each $i, j$ pair, let $\min _{i j}(b)$ be the minimum value of $f_{i j}\left(b^{\prime}\right)$ on any assignment $b^{\prime} \in\{0,1\}^{n}$ such that $b^{\prime} \succeq b$, and let $\max _{i j}(b)$ be the maximum value. Let $R_{\max (i j)}=$ $\max _{i j}(*, \ldots, *)$, and let $R_{\min (i j)}=\min _{i j}(*, \ldots, *)$.

Let $g_{<}^{(i j)}:\{0,1, *\}^{n} \rightarrow \mathbb{Z}_{\geq 0}$ be defined as follows. If $R_{\max (i j)} \leq 0$, then $g_{<}^{(i j)}(b)=0$ for all $b \in\{0,1, *\}^{n}$ and $Q_{<}^{(i j)}=0$. Otherwise, for $b \in\{0,1, *\}^{n}, \operatorname{let} g_{<}^{(i j)}(b)=\min \left\{R_{\max (i j)}, R_{\max (i j)}-\right.$ $\left.\max _{i j}(b)\right\}$ and $Q_{<}^{(i j)}=R_{\max (i j)}$. It follows that for $b \in\{0,1, *\}^{n}, f_{i}\left(b^{\prime}\right) \leq f_{j}\left(b^{\prime}\right)$ for all extensions $b^{\prime} \sim b$ if and only if $g_{<}^{(i j)}(b)=Q_{<}^{(i j)}$.

We define $g_{>}^{(i j)}$ and $Q_{>}^{(i j)}$ symmetrically so that $f_{i}\left(b^{\prime}\right) \geq f_{j}\left(b^{\prime}\right)$ for all extensions $b^{\prime} \sim b$ if and only if $g_{>}^{(i j)}(b)=Q_{>}^{(i j)}$.

We apply the disjunctive construction of Lemma 1 to combine $g_{>}^{(i j)}$ and $g_{<}^{(i j)}$ and their associated goal values. Let the resulting new utility function be $g^{(i j)}$, and let its goal value be $Q^{(i j)}$. Then $Q^{(i j)}$ is $O\left(D^{2}\right)$, where $D$ is the sum of the magnitudes of the coefficients in $f_{i j}$.

Using the conjunctive construction of Lemma 1 to combine the $g^{(i j)}$, we get our final utility function $g=\sum_{i<j} g^{(i j)}$ with goal value $Q=\sum_{i<j} Q^{(i j)}$.

We now show that achieving the goal utility $Q$ is equivalent to having enough information to do the ranking. Until the goal value is reached, there is still a pair $i, j$ such that it remains possible that $f_{i}(x)>f_{j}(x)$ (under one setting of the untested variables) and it remains possible that $f_{j}(x)<f_{i}(x)$ (under another setting). In this situation, we do not have enough information to output a ranking we know to be valid.

Once $g(b)=Q$, the situation changes. For each $i, j$ such that $f_{i}(x)<f_{j}(x)$, we know that $f_{i}(x) \leq f_{j}(x)$. Similarly, if $f_{i}(x)>f_{j}(x)$, then at goal utility $Q$, we know that $f_{i}(x) \geq f_{j}(x)$. If $f_{i}(x)=f_{j}(x)$ at goal utility $Q$, we may only know that $f_{i}(x) \geq f_{j}(x)$ or that $f_{i}(x) \leq f_{j}(x)$. We build a valid ranking from this knowledge as follows. If there exists an $i$ such that we know that $f_{i}(x) \leq f_{j}(x)$ for all $j \neq i$, then we place $f_{i}(x)$ first in our ranking and recursively rank the other elements. Otherwise, we can easily 
find a "directed cycle"-that is, a sequence $i_{1}, \ldots, i_{m}, m \geq 2$, such that we know that $f_{i_{1}}(x) \leq f_{i_{2}}(x) \leq \cdots \leq f_{i_{m}}(x)$ and $f_{i_{m}}(x) \leq f_{i_{1}}(x)$. It follows that $f_{i_{1}}(x)=\cdots=f_{i_{m}}(x)$. In this case, we can delete $f_{i_{2}}, \ldots, f_{i_{m}}$, recursively rank $f_{i_{1}}$ and the remaining $f_{i}$, and then insert $f_{i_{2}}, \ldots, f_{m}$ into the ranking next to $f_{i_{1}}$.

Applying the adaptive greedy algorithm to solve the SSSC problem for $g$, the theorem follows from the $(\ln Q+1)$ approximation bound for this algorithm and the fact that $Q=O\left(D_{\max }^{2} m^{2}\right)$.

\section{APPENDIX}

\section{A. TABLE OF NOTATIONS}

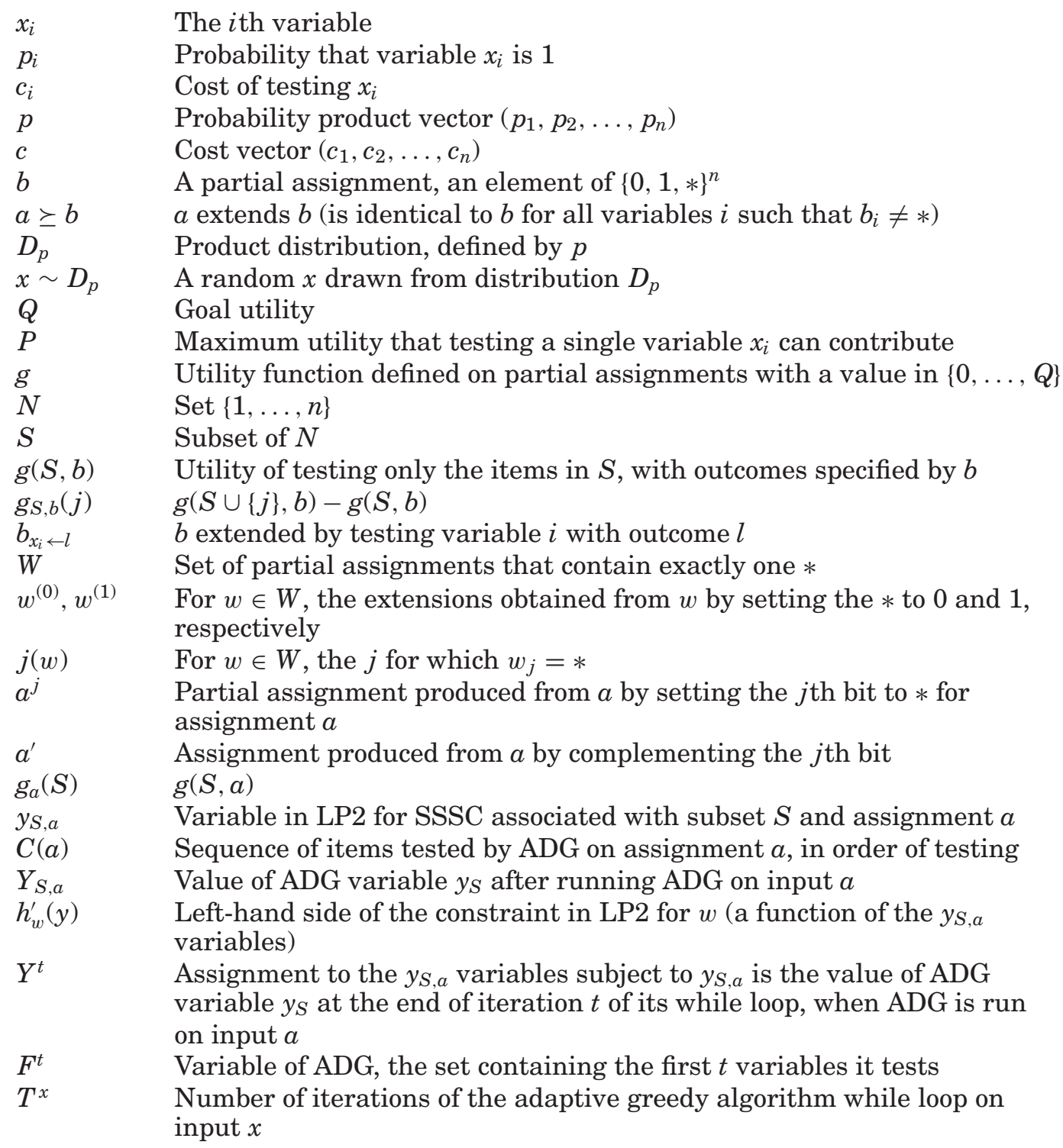




$\begin{array}{ll}b_{x}^{t} & \begin{array}{l}\text { Value of } b \text { on input } x \text { after the } t \text { th iteration of the loop of the adaptive } \\ \text { greedy algorithm on } x\end{array} \\ Y^{x} & \text { Assignment to the LP2 variables used in the analysis of the new bou } \\ & \text { for the adaptive greedy algorithm } \\ q^{x}(y) & \sum_{S \subset N}(Q-g(S, x)) y_{S, x} \\ g_{b}(i, l) & \begin{array}{l}\text { Equals increase in utility of test on } i \text { with outcome } l \text { if } i \text { was not yet } \\ \text { tested (else equals } 0), \text { used in analysis of AG }\end{array} \\ g(j) & \text { Equals } \max _{l \in\{0,1\}} g_{r}(j, l), \text { where } r=(*, \ldots, *), \text { used in analysis of the } \\ & \text { adaptive greedy algorithm }\end{array}$

\section{ACKNOWLEDGMENTS}

We thank Tonguç Ünlüyurt and Sarah Allen for helpful feedback, Patrick Lin for proofreading and improving the notation, and Sarah Allen for a notation summary. We thank anonymous referees for helpful suggestions, such as applying our techniques to stochastic min-knapsack, and suggesting a way to simplify our original analysis of ADG. Alina Ene told us about the connection of the work of Im et al. to the stochastic min-knapsack problem. Lisa Hellerstein would like to thank Endre Boros, Kazuhisa Makino, and Vladimir Gurvich for a stimulating discussion at RUTCOR.

\section{REFERENCES}

M. Adler and B. Heeringa. 2012. Approximating optimal binary decision trees. Algorithmica 62, $1112-1121$.

S. R. Allen, L. Hellerstein, D. Kletenik, and T. Ünlüyurt. 2013. Evaluation of DNF formulas. arXiv:1310. 3673.

A. Bar-Noy, M. Bellare, M. M. Halldórsson, H. Shachnai, and T. Tamir. 1998. On chromatic sums and distributed resource allocation. Information and Computation 140, 183-202.

G. Bellala, S. Bhavnani, and C. Scott. 2012. Group-based active query selection for rapid diagnosis in timecritical situations. IEEE Transactions on Information Theory 58, 1, 459-478.

Y. Ben-Dov. 1981. Optimal testing procedures for special structures of coherent systems. Management Science 27, 12, 1410-1420.

P. Beraldi and A. Ruszczynski. 2002. The probabilistic set-covering problem. Operations Research 50, 956967.

A. Bhalgat. 2011. A $(2+\epsilon)$-approximation algorithm for the stochastic knapsack problem. Unpublished Manuscript.

A. Bhalgat, A. Goel, and S. Khanna. 2011. Improved approximation results for stochastic knapsack problems. In Proceedings of the 22nd Annual ACM-SIAM Symposium on Discrete Algorithms. 16471665.

E. Boros and T. Ünlüyurt. 1999. Diagnosing double regular systems. Annals of Mathematics and Artificial Intelligence 26, 171-191.

E. Boros and T. Ünlüyurt. 2000. Sequential testing of series-parallel systems of small depth. In Computing Tools for Modeling, Optimization and Simulation. Springer, 39-74.

T. Carnes and D. Shmoys. 2008. Primal-dual schema for capacitated covering problems. In Proceedings of the 13th International Conference on Integer Programming and Combinatorial Optimization (IPCO'08). 288-302.

R. Carr, L. Fleischer, V. Leung, and C. Phillips. 2000. Strengthening integrality gaps for capacitated network design and covering problems. In Proceedings of the 11th Annual ACM-SIAM Symposium on Discrete Algorithms. 106-115.

M.-F. Chang, W. Shi, and W. K. Fuchs. 1990. Optimal diagnosis procedures for $k$-out-of- $n$ structures. IEEE Transactions on Computers 39, 559-564.

M. Charikar, R. Fagin, V. Guruswami, J. M. Kleinberg, P. Raghavan, and A. Sahai. 2002. Query strategies for priced information. Journal of Computer and Systems Sciences 64, 785-819.

F. Cicalese, E. Laber, and A. M. Saettler. 2013. Decision trees for the efficient evaluation of discrete functions: Worst case and expected case analysis. arXiv 1309.2796.

L. Cox, Y. Qiu, and W. Kuehner. 1989. Heuristic least-cost computation of discrete classification functions with uncertain argument values. Annals of Operations Research 21, 1-29. 
B. Dean, M. Goemans, and J. Vondrák. 2004. Approximating the stochastic knapsack problem: The benefit of adaptivity. In Proceedings of the 45th Symposium on Foundations of Computer Science (FOCS'04). 208-217.

C. Derman, C. Lieberman, and S. Ross. 1978. A renewal decision problem. Management Science 24, 5, $554-561$.

A. Deshpande and L. Hellerstein. 2008. Flow algorithms for parallel query optimization. In Proceedings of the 24th International Conference on Data Engineering (ICDE'08). 754-763.

U. Feige, L. Lovász, and P. Tetali. 2002. Approximating min-sum set cover. In Proceedings of the 5th International Workshop on Approximation Algorithms for Combinatorial Optimization (APPROX'02). 94-107.

A. Fiat and D. Pechyony. 2004. Decision trees: More theoretical justification for practical algorithms. In Proceedings of the 15th International Conference on Algorithmic Learning Theory (ALT'04).

T. Fujito. 1999. On approximation of the submodular set cover problem. Operations Research Letters 25, 4 , $169-174$.

T. Fujito. 2000. Approximation algorithms for submodular set cover with applications. IEICE Transactions on Information and Systems 83, 480-487.

M. Garey. 1973. Optimal task scheduling with precedence constraints. Discrete Mathematics 4, 37-56.

M. X. Goemans and J. Vondrák. 2006. Stochastic covering and adaptivity. In Proceedings of the 7th Latin American Symposium on Theoretical Informatics (LATIN'06). 532-543.

D. Golovin and A. Krause. 2011. Adaptive submodularity: Theory and applications in active learning and stochastic optimization. Journal of Artificial Intelligence Research 42, 427-486.

D. Golovin, A. Krause, and D. Ray. 2010. Near-optimal Bayesian active learning with noisy observations. In Proceedings of the 24th Annual Conference on Neural Information Processing Systems (NIPS'10). 766-774.

R. Greiner, R. Hayward, M. Jankowska, and M. Molloy. 2006. Finding optimal satisficing strategies for and-or trees. Artificial Intelligence 170, 19-58.

R. Greiner, R. Hayward, and M. Molloy. 2002. Optimal depth-first strategies for and-or trees. In Proceedings of the 18th National Conference on Artificial Intelligence and the 14th Conference on Innovative Applications of Artificial Intelligence. 725-730.

D. Guijarro, V. Lavín, and V. Raghavan. 2006. Exact learning when irrelevant variables abound. In Proceedings of the 4th European Conference on Computational Learning Theory (EuroCOLT'99). 91-100.

A. Guillory and J. Bilmes. 2011. Simultaneous learning and covering with adversarial noise. In Proceedings of the 28th International Conference on Machine Learning (ICML'11). 369-376.

X. Han and K. Makino. 2010. Online minimization knapsack problem. In Approximation and Online Algorithms. Lecture Notes in Computer Science, Vol. 5893. Springer, 182-193.

J. Hastad. 1994. On the size of weights for threshold gates. SIAM Journal on Discrete Mathematics 7, 3, 484-492.

T. Ibaraki and T. Kameda. 1984. On the optimal nesting order for computing $n$-relational joins. ACM Transactions on Database Systems 9, 482-502.

S. Im and V. Nagarajan. 2011. Minimum latency submodular cover in metrics. arXiv: 1110.2207.

S. Im, V. Nagarajan, and R. van der Zwaan. 2012. Minimum latency submodular cover. In Proceedings of the 39th International Colloquium on Automata, Languages, and Programming (ICALP'12). 485-497.

S. Iwata and K. Nagano. 2009. Submodular function minimization under covering constraints. In Proceedings of the 50th IEEE Symposium on Foundations of Computer Science (FOCS'09). 671-680.

H. Kaplan, E. Kushilevitz, and Y. Mansour. 2005. Learning with attribute costs. In Proceedings of the Symposium on the Theory of Computing. 356-365.

R. Krishnamurthy, H. Boral, and C. Zaniolo. 1986. Optimization of nonrecursive queries. In Proceedings of the 12th International Conference on Very Large Data Bases (VLDB'86). 128-137.

Z. Liu, S. Parthasarathy, A. Ranganathan, and H. Yang. 2008. Near-optimal algorithms for shared filter evaluation in data stream systems. In Proceedings of the 28th ACM SIGMOD International Conference on Management of Data.

M. Moshkov. 2003. Approximate algorithm for minimization of decision tree depth. In Rough Sets, Fuzzy Sets, Data Mining, and Granular Computing. Lecture Notes in Computer Science, Vol. 2639. Springer, 611-614.

M. Moshkov and I. Chikalov. 1997. Bounds on average weighted depth of decision trees. Fundamenta Informaticae 31, 145-156.

K. Munagala, S. Babu, R. Motwani, and J. Widom. 2005. The pipelined set cover problem. In Proceedings of the 10th International Conference on Database Theory (ICDT'05). 83-98. 
S. Nijssen and E. Fromont. 2007. Mining optimal decision trees from itemset lattices. In Proceedings of the 13th ACM SIGKDD International Conference on Knowledge Discovery and Data Mining (KDD'07). 530-539.

S. Salloum. 1979. Optimal Testing Algorithms for Symmetric Coherent Systems. Ph.D. Dissertation. University of Southern California.

S. Salloum and M. Breuer. 1984. An optimum testing algorithm for some symmetric coherent systems. Journal of Mathematical Analysis and Applications 101, 170-194.

U. Srivastava, K. Munagala, J. Widom, and R. Motwani. 2006. Query optimization over Web services. In Proceedings of the 32nd International Conference on Very Large Data Bases (VLDB'06). 355-366.

T. Ünlüyurt. 2004. Sequential testing of complex systems: A review. Discrete Applied Mathematics 142, 189-205.

L. Wolsey. 1982. An analysis of the greedy algorithm for the submodular set covering problem. Combinatorica 2, 385-393.

Received December 2014; revised January 2016; accepted January 2016 